\title{
评 述＼cjkstart庆祝蔡启瑞院士百岁华诞专题
}

\section{煤炭间接液化：从基础到工业化}

\author{
相宏伟 ${ }^{(12)}$ ，杨勇 ${ }^{(1)}$ ，李永旺 ${ }^{(1)}$ *
}

(1) 中国科学院山西煤炭化学研究所煤转化国家重点实验室, 太原 030001

(2) 中科合成油技术有限公司煤基清洁燃料国家能源研究中心, 北京 101407

*通讯作者, E-mail: ywl@sxicc.ac.cn

收稿日期: 2014-08-04; 接受日期: 2014-08-25; 网络版发表日期: 2014-11-21 doi: 10.1360/N032014-00218

摘要中国需要自主发展煤炭间接液化工业化技术, 以缓解油品供应的紧张局面, 保障经 济的可持续发展. 近年来中国成功地进行了煤炭间接液化示范厂的运行, 掌握了成熟可靠的 费托合成催化剂技术和大型合成反应器技术, 正在设计和建设百万吨级合成油商业厂. 本文 简要介绍了国内外煤炭间接液化技术发展状态，评述了我国煤炭间接液化技术在费托合成 反应机理、催化剂研制、反应动力学、反应器设计、系统工艺集成、油品加工等方面从基础 到工程技术的研究进展, 分析了我国建设百万吨级煤炭间接液化商业厂需要解决的关键基 础和工程技术问题, 并对我国未来煤制油产业化发展的前景以及所面临的挑战与对策进行 了展望.

\section{1 引言}

油品是国家工业、经济和社会赖以运行的血液和 动力源泉, 是社会发展和人类生存不可或缺的能源 资源. 2013 年我国石油消费量达到 4.98 亿吨, 进口量 达到 2.82 亿吨, 对外依存度达到 $58.1 \%{ }^{[1]}$, 已逼近我 国《能源发展“十二五”规划》61\%的红线 ${ }^{[2]}$, 预计 2020 年我国石油消费量将达到 6 亿吨, 对外依存度将达到 $68 \%$. 目前我国自有石油产能已达到顶峰( 2.0 亿吨/ 年), 新增石油探明储量有限, 而目前我国石油进口 主要来自于局势动荡或具有潜在不稳定因素的中东、 北非、南美、西亚和俄罗斯等地区或国家 ${ }^{[3]}$. 在我国 海陆石油输运路径均不能得到安全有效的控制, 且 新的进口渠道拓展并不十分明朗的情况下, 我国油 品供应的紧张局势将愈加凸显, 这将成为制约我国 经济发展和威胁我国能源安全的重大问题.

我国一次能源以煤为主, 2013 年煤炭在一次能 源消费中占 $67.5 \% \%^{[4]}$, 虽然风能、太阳能、生物质能、
核能等在一定程度上可以缓解能源紧张局面, 但预 计到 2030 年之前我国以煤炭为主要能源的格局不会 改变. 我国煤炭资源相对较为丰富, 国土资源部最近 的评估表明, 我国 $2000 \mathrm{~m}$ 以浅煤炭资源总量 5.9 万亿 吨, 其中探获资源储量 2.02 万亿吨 ${ }^{[5]}$; 能满足现行开 采技术条件的基础储量为 3189.6 亿吨 ${ }^{[6]} .2013$ 年我国 原煤产量为 37 亿吨, 煤炭消费量为 36.1 亿吨, 占全 球煤炭消费量的一半 ${ }^{[7]}$. 按照目前我国煤炭消费速度, 我国煤炭至少可供开采 80 年以上. 我国石油地质资 源量为 1037 亿吨, 可采资源量为 264 亿吨 ${ }^{[8]}$, 考虑到 经济可采情况还可供开采 40 年左右. 在新能源还难 以为续、传统石油储量有限、油品供不应求的时期, 面对亿吨级油品的供应缺口, 近中期内(30 50 年)只 有煤炭资源能够在量级上相匹配, 因此以煤为原料, 科学有序、在技术示范成熟的基础上发展煤制油产业 已成为我国破除能源困局的现实选择之一 ${ }^{[9 \sim 11]}$.

煤制油技术主要有两条途径: 一是煤炭直接液 化, 即将煤与重油混合的煤油浆在高温 $\left(400 \sim 700^{\circ} \mathrm{C}\right)$ 
和高压(15.0 30.0 MPa)下加氢, 获得初原油, 再加氢 精制生产柴油、汽油、石脑油等产品; 二是煤炭间接 液化, 即煤首先通过氧气、水蒸气被气化制得合成气 $\left(\mathrm{H}_{2}\right.$ 与 $\mathrm{CO}$ 的混合气), 合成气再在催化剂作用下反应 生成液态烃、蜡、气态轻烃和部分有机含氧化合物, 该反应被称为费托合成(F-T 合成)反应，中间产物经 加工后可获得柴油、汽油、煤油、石脑油、液化石油 气(LPG)及精细化学品等产品. 煤炭间接液化过程中 的关键步骤——费托合成反应可在温和条件下 $\left(200 \sim 300^{\circ} \mathrm{C}, 2.0 \sim 3.0 \mathrm{MPa}\right)$ 进行, 生产装备易于大型 化和国产化, 且经煤炭间接液化工艺可生产出的无 硫、无氮、低芳、高十六烷值(> 70)的清洁油品, 使得 煤炭间接液化成为发展我国煤制油产业优先选择的 技术路线之一。

一个国家煤制油工业技术的开发总是受到战争、 经济发展、石油价格上涨、国际原油封锁、能源战略 安全等因素的影响而发展或停顿. 1923 年 Fischer 和 Tropsch 发现合成气在铁催化剂上可生成液体燃料的 反应 ${ }^{[12]}$, 至今煤炭间接液化工业化已经历了 90 多年 的历史, 早期(1934 1945 年)德国因战争的需要曾建 有费托法煤制油厂, 后该技术转移到南非, 目前国际 上仅有南非 Sasol 公司和荷兰 Shell 公司拥有以费托合 成为核心的煤制油和天然气制油的工业化技术 ${ }^{[11,13]}$. 中国最早从 1941 年开始进行费托法煤制油技术的研 究和装置建设 ${ }^{[14]}$. 从现代煤化工角度看, 中国煤炭间 接液化技术的研究自 1997 年才开始真正起步, 2009 年内蒙古伊泰和山西潞安两个 16 万吨/年合成油示范 厂投产出油, 随后实现了“安稳长满优”的工业生产, 标志着我国已掌握了先进可靠的煤炭间接液化工业 技术 ${ }^{[15 ~ 17]}$. 利用国外现有的煤炭间接液化技术发展 我国煤制油产业，技术专利费高，代价大，且受控于 人, 自主开发煤炭间接液化工业技术是建设我国煤 制油产业的根本出路. 2011 年神华宁煤集团决定采用 我国自主开发的高温浆态床煤制油工艺技术建设国 内最大的 400 万吨/年煤制油装置, 摈弃谈判近十年 的南非 Sasol 公司技术, 标志着我国进入了科学有序 大规模自主发展煤制油工业的阶段 ${ }^{[18]}$.

煤炭间接液化工业过程的开发目标是提高过程 的能量转化效率和技术经济性, 制约效率提升的主 要难点和核心技术为: (1) 较高操作温度下, 高合成 气转化和产油能力、高 $\mathrm{C}_{3+}$ 烃类产品选择性的费托合 成催化剂研发及制备技术; (2) 可大规模化、操控连
续稳定、反应热回收效率高且能高效分离产品的合成 反应器技术; (3) 以费托合成为核心的、兼顾上下游 工艺单元的全系统优化集成技术; (4) 满足严苛条件 的先进的节水和环境排放技术. 本文评述了国内外 煤炭间接液化技术的发展, 介绍了我国煤炭间接液 化技术从基础研究到工业示范的研究进展和所取得 的成就，探讨了我国建设百万吨级煤制油装置存在 的关键问题与可能的解决对策, 并对今后发展我国 煤制油工业进行了展望.

\section{2 国内外煤炭间接液化技术发展状况}

\section{1 国外煤炭间接液化技术发展}

煤炭间接液化的费托合成油技术最早可追溯到 20 世纪 20 年代, 1923 年德国科学家 Fischer 和 Tropsch 发现合成气在铁催化剂上可生成液体燃料的反应, 该 方法称为费托合成法 ${ }^{[19]} .1934$ 年德国开始建造以煤为 原料的费托合成油厂，使用常压钴基固定床费托合成 技术, 1936 年投产. 1936 1945 年间, 德国共建有 9 个 费托合成油厂，总产量达 67 万吨/年，同期，法、日、 中、美等国也建有 7 套以煤为原料的费托合成油厂，总 产能达 69 万吨/年. 二战后, 由于石油工业的兴起, 廉 价油品的生产，致使费托合成油装置纷纷关闭 ${ }^{[11,13]}$.

南非拥有丰富的煤炭资源，但缺少石油资源，由 于南非实行种族隔离制度导致国际上对其进行石油 禁运政策, 由此被迫发展煤制油工业. 从 20 世纪 50 年代开始南非引进了德国和美国早期的费托合成油 技术，成立了南非 Sasol 公司. 1955 年建成 Sasol I 厂, 使用低温固定床铁基催化剂费托合成技术, 生产柴 油和费托蜡 ${ }^{[20,21]}$. 1955 年后开发出高温费托合成循 环流化床反应器, 采用熔铁催化剂, 主要生产汽油和 低碳烯烃，单台产能达到 1500 桶/天，1980 年、1982 年 Sasol 公司先后建成 Sasol II 厂和 Sasol III 厂, 开发 出单台产能达 6500 桶/天的循环流化床合成反应器, 1985 年 Sasol 公司开发出固定流化床合成工艺, 1995 年直径 $8 \mathrm{~m}$ 的固定流化床反应器装置开车成功, 单台 产能 11000 桶/天, 1996 1999 年, 又开发出了直径 $10.7 \mathrm{~m}$ 、单台产能达 20000 桶/天的固定流化床反应 器 ${ }^{[20,21]} .1995$ 年建成投产了直径 $5 \mathrm{~m}$ 、单台产能为 2500 桶/天的浆态床合成油工业装置, 使用沉淀型微 球状铁基催化剂，形成了成熟的低温 $\left(200 \sim 250^{\circ} \mathrm{C}\right)$ 浆 态床合成油技术，主要生产柴油、石脑油和费托蜡等 
产品 ${ }^{[20,21]}$. 近年来 Sasol 公司又开发出了钴基低温浆 态床合成工艺 $\left(200 \sim 250^{\circ} \mathrm{C}\right)$, 设计了直径 $9.6 \mathrm{~m}$ 、单台 产能达 60 80 万吨/年浆态床合成反应器, 2006 年在 卡塔尔建成投产了 140 万吨/年天然气制油(GTL)装置, 运行时遇到催化剂流失和蜡催化剂分离问题, 2008 年 实现正常运行. 2013 年 Sasol 公司也使用钴基低温浆 态床合成工艺在尼日利亚建成投产了 140 万吨/年 GTL 装置 ${ }^{[22]}$; 此外 Sasol 公司将在美国建设 400 万吨/ 年 GTL 装置, 预计 2014 年完成设计, 2018 年建成投 ${ }^{\text {产 }}{ }^{[23]}$. Sasol 公司钴基低温浆态床合成工艺至今并未 用于煤制油领域, 该工艺催化剂价格高, 要求催化剂 寿命长, 须避免催化剂磨损带来钴的损失, 产生的大 量低压蒸汽难以有效利用. 1995 年前 Sasol 公司主要 依靠政府补贴维持运行, 1995 年后开始不依赖于政府 补贴而获得盈利. 目前南非 Sasol 公司是世界上最大 的综合性煤化工企业, 年耗煤 5000 万吨以上, 以煤 间接液化技术为核心, 年产油品和化学品 850 万吨, 其中油品 600 万吨, 生产的合成油供应南非 $30 \%$ 的油 品市场, 并提供 300 多种化学品. Sasol 公司 2013 年 销售额达到 205 亿美元, 实现利润 46 亿美元. 南非近 60 年技术发展的实践证明煤制油产业是可以赢利的, 技术的不断进步是突破煤制油技术经济瓶颈的关键 所在. 近年来 Sasol 公司一直尝试将其费托合成技术 拓展到中国和印度煤制油市场.

除南非 Sasol 公司外, 荷兰 Shell 公司也拥有工业 化的费托合成技术, 1993 年在马来西亚建成一套天然 气制中间馏分油的 50 万吨/年的装置, 采用固定床钴 基催化剂合成技术, 反应器直径为 $7 \mathrm{~m}$, 单台产能为 3000 桶/天 ${ }^{[24]}$. 近年来该装置扩建为 75 万吨/年, 单台 反应器产能提高到 8000 桶/天. 2011 年 Shell 公司在卡 塔尔建成投产了 150 万吨/年的 GTL 装置(一期工程), 2012 年 600 万吨/年全套 GTL 装置投产 (140000 桶/

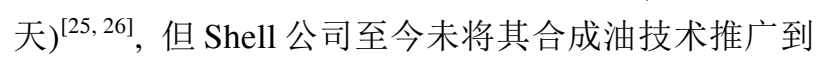
煤制油领域. 到目前为止, 国际上仅有南非 Sasol 公 司和荷兰 Shell 公司拥有费托合成油工业技术, 其他 如美国 Syntroleum 公司、美国 Exxon-Mobil 公司、美 国 ConocoPhillips 公司、英国 BP-Amoco 公司、丹麦 Tops申e 公司等也在开发费托合成技术 ${ }^{[11,22,27]}$, 但均 未实现商业化.

\section{2 国内煤炭间接液化技术发展}

我国曾是世界上较早拥有煤制油工厂的国家之
一. 1937 年日军在锦州石油六厂引进德国常压钴基固 定床费托合成技术建设煤制油厂, 1943 年运行并生产 原油 100 吨/年, 1945 年日本战败后停产. 1939 年当中 日战争进入最困难的时候, 日军对中国进行全面物资 封锁, 中国军队所需燃油进口非常困难时, 国民政府 决定学习德国技术, 进行煤制油技术研究 ${ }^{[14,28]} .1941$ 年赵宗燠 ${ }^{[14]}$ 开始在重庆建设费托合成法的煤制油厂, 在小型试验装置上产出了油, 1950 年赵宗燠等 ${ }^{[14]}$ 接管 并恢复扩建了锦州煤制油装置, 1951 年生产出油, 1959 年产量最高时达 4.7 万吨/年. 同期的 1953 年中 国科学院大连石油研究所(现中国科学院大连化学物 理研究所 $)^{[28]}$ 进行了 4500 吨/年铁催化剂流化床合成 油中试试验, 但技术未过关(催化剂磨损、黏结). 这 时因大庆油田的发现，中国放弃了当时被称为“人造 石油”之梦的追求, 我国煤制油装置全部关闭, 技术 开发终止. 1980 年中国基于能源安全与技术储备的考 虑, 恢复并开始了第二次煤制油技术的开发, 中国科 学院山西煤碳化学研究所提出有别于南非技术的、将 传统的费托合成与沸石分子篮相结合的固定床两段 合成工艺(MFT工艺) ${ }^{[29,30]}, 1989$ 年完成 100 吨/年工业 中试, 1993 1994 年完成了 2000 吨/年工业试验, 产出 合格的 90 号汽油, 因当时油价过低, 技术经济性很 难过关, 且后续支持经费难以到位, 而重新陷入煤制 油技术开发的低谷.

1997 年中国科学院山西煤碳化学研究所李永旺 课题组调阅了我国经历的全部的煤制油试验资料, 分析了中国煤炭和石油资源的状况、国际油价的走 势、中国经济的发展速度和国民可以买得起轿车的时 间节点, 认为煤制油技术经济性如果得到持续的改 进, 预测在 2005 2008 年煤制油技术经济性与油价的 关系就会出现转折点, 那么大规模建设煤制油厂的 时机就会来临, 决定将主攻方向由原来的固定床合 成工艺转变为更为先进的浆态床合成工艺 ${ }^{[31]}$. 1998 2000 年在实验室成功地研制出了高性能的低 温浆态床费托合成铁基催化剂, 同时蜡催化剂分离 技术获得重大进展, 浆态床煤制油工艺的技术经济 性瓶颈得到突破 ${ }^{[32,33]}$. 2001 年建起千吨级浆态床合 成工业中试装置, 2004 年开发出与国外水平相当的低 温浆态床煤制油技术, 随后针对低温合成过程较低 能效的缺陷，提出高温浆态床间接液化工艺技术的 概念 ${ }^{[15 ~ 17],} 2006$ 年成立了中科合成油技术有限公司, 2006 2008 年又成功开发出国际上最为先进的高温 
浆态床煤制油工艺及催化剂成套技术, 2008 年建成投 产了 1500 吨/年费托合成催化剂厂，2009年建成投产 了两个 16 万吨/年煤炭间接液化示范厂, 实现了“安 稳长满优” 的工业生产 ${ }^{[34]}$. 同期上海充矿能源科技研 发有限公司也分别完成了 5000 吨/年的低温浆态床合 成和高温固定流化床合成的工业中试试验 ${ }^{[35]} .2011$ 年神华宁煤集团决定采用我国自主开发的中科高温 浆态床煤制油工艺技术建设国内最大的 400 万吨/年 煤制油商业装置, 结束了我国政府与南非 Sasol 公司 近 10 年的引进技术谈判 ${ }^{[18]}$, 目前自主技术正在实施 近 1600 万吨/年产能的煤炭间接液化商业装置的建设, 由此我国开始进入了科学有序大规模发展煤制油工 业的时期.

\section{3 催化与工程技术基础}

煤炭间接液化是一个涉及输煤备煤、煤气化、合 成气净化与变换、费托合成、油品加工、大型合成反 应器的设计制造与控制、尾气转化与发电、产品分离、 环保处理等的复杂的系统工程. 提高煤炭间接液化 过程的能量利用效率需要全系统的集成优化, 这依 赖于合成工艺的创新、详细机理的费托合成反应动力 学模型和反应器模型的建立, 以及系统工艺模拟软 件的构建. 关键的费托合成铁基催化剂在反应过程 中表现为复杂的多重铁碳化物和铁物相结构, 且产 物复杂 $\left(\mathrm{C}_{1} \sim \mathrm{C}_{100}\right.$ 的烃和副产醇、醛、酸、酯等), 从原 子/分子尺度上理解费托反应机理和活性相的稳定结 构与晶面取向, 对研制高活性、运行稳定和可以有效 控制烃产物选择性的催化剂至关重要. 在浆态床合 成反应器技术方面, 国外在关键的内构件和蜡催化 剂分离技术方面一直很保密, 真正工业应用的技术 在公开的国际专利中难以获取, 需要解决高温 $\left(260 \sim 290^{\circ} \mathrm{C}\right)$ 操作下的费托合成强放热控制与热量高 效回收、蜡催化剂分离的技术难题. 针对费托合成中 间产物无硫、无氮、低芳烃且以直链烷烯烃为主的特 点需要研制非硫态化的油品加工催化剂, 优化油品 工艺路线与方案, 以生产满足市场需求的高品质的 柴油、汽油、航煤等产品.

\section{1 高温浆态床费托合成工艺}

国际上南非 Sasol 公司采用低温浆态床费托合成 工艺(微球状铁或钴基催化剂, 反应温度 200 250 ${ }^{\circ} \mathrm{C}$ )、
低温固定床费托合成工艺(沉淀型铁基催化剂, 反应 温度 $200 \sim 250^{\circ} \mathrm{C}$ ) 和高温循环流化床和固定流化床费 托合成工艺 (熔铁催化剂, 反应温度 $300 \sim 340^{\circ} \mathrm{C}$ ), 荷 兰 Shell 公司采用低温固定床合成工艺(钴基催化剂, 反应温度 $\left.190 \sim 230^{\circ} \mathrm{C}\right)$. 低温浆态床和固定床合成工 艺 $\left(190 \sim 250^{\circ} \mathrm{C}\right)$ 虽能保证较低的甲烷选择性和高的柴 油收率, 但大量放出的反应热副产的蒸汽( $0.5 \sim 0.8$ $\mathrm{MPa}$ 品位低, 难以在全厂实现平衡利用, 整体能量 利用效率为 37\% 38\%. 高温流化床合成工艺(300 $\left.340^{\circ} \mathrm{C}\right)$ 可将反应热转化为高品位的蒸汽(3.5 5.0 MPa), 这部分热量可以通过全厂能量平衡和发电获得充分 利用, 但过高的反应温度导致产物中甲烷选择性过 高(10\% 12\%), 油品收率降低, 同时尾气转化负荷增 加, 又降低了能量利用效率, 该工艺整体能效为 38\% 39\%.

李永旺等 ${ }^{[15 ~ 17]}$ 在国际上首次提出高温浆态床费 托合成工艺概念, 即将浆态床费托合成反应温度由 $200 \sim 250^{\circ} \mathrm{C}$ 提高到 $260 \sim 290^{\circ} \mathrm{C}$, 副产高品位蒸汽 (2.0 3.0 MPa), 联产发电, 可有效地平衡全系统的热 量, 克服低温浆态床工艺的低品位蒸汽难以高效利 用的缺点, 从而提升煤间接液化过程的整体能量利 用效率. 反应温度的提高有利于提高费托合成过程 副产蒸汽的压力等级, 进而提高蒸汽利用效率, 但受 制于反应动力学和热力学控制, 催化剂的产物选择 性有可能会严重轻质化, 甲烷选择性升高; 同时催化 剂会过度碳化而降低抗磨损性能, 增加浆态床合成 反应器的过滤负荷和难度. 另外反应温度的提高, 也 会增加浆态床合成反应器的液泛风险和控制难度. 如何研制出在较高温下获得稳定的物相结构、高的合 成气转化能力、低的甲烷和有机含氧化物选择性、高 的有效烃 $\left(\mathrm{C}_{3+}\right.$ 烃)的选择性和良好的抗磨损性能的催 化剂以及如何设计和控制适合高温运行的浆态床合 成反应器成为技术关键.

\section{2 费托合成反应机理和详细机理动力学}

费托合成反应的产物非常复杂, 主要包括烷烃、 烯烃、少量的有机含氧化合物如醇、醛、酮、酸、酯 等, 还有副产物 $\mathrm{H}_{2} \mathrm{O}$ 和 $\mathrm{CO}_{2}$. 费托合成反应机理极其 复杂, 一般假定烃生成为聚合反应, 经历链引发、链 增长、链终止/脱附等三个阶段. Fischer 和 Tropsch ${ }^{[13,19]}$ 最早提出了表面碳化物机理, 认为 $\mathrm{CO}$ 首先在催化剂 
表面吸附解离为活性金属碳化物物种, 然后与 $\mathrm{H}_{2}$ 反 应形成亚甲基 $\left(-\mathrm{CH}_{2}-\right)$ 中间体，再进一步聚合生成烷 烃和烯烃. 该机理可以很好地描述烃类产物生成的 分布规律, 但无法解释支链产物和有机含氧化合物 的生成. 后有人在碳化物机理基础上提出了烷基化 机 理 ${ }^{[36]}$, 认为表面碳物种 $(\mathrm{CM})$ 依次加氢生成的 $\mathrm{CHM} 、 \mathrm{CH}_{2} \mathrm{M} 、 \mathrm{CH}_{3} \mathrm{M}$ 等表面物种, 这些表面物种可 以通过烷基键合反应生成链终止产物 $\mathrm{C}_{n} \mathrm{H}_{n+1} \mathrm{M}$, 然后 通过加氢生成 $n$-烷烃和 $\beta-\mathrm{H}$ 消除生成 $\alpha$-烯烃. 该机理 不包含生成支链烃, 支链烃的生成部分源于烯烃再 吸附, Lee 等 ${ }^{[37]}$ 和 Anderson ${ }^{[38]}$ 分别提出了烯烃的再吸 附生成支链烃的反应机理. 为解释有机含氧化合物 的生成, Storch 等 ${ }^{[39]}$ 和 Eidus ${ }^{[40]}$ 分别提出了烯醇机理, 认为 $\mathrm{COM}$ 和 $\mathrm{HM}$ 反应生产表面烯醇中间物种, 两个 $\mathrm{M}=\mathrm{CHOH}$ 物种缩合形成 $\mathrm{CHCOH}$ 中间体, 进一步加 氢形成烃和有机含氧化合物, 但是烯醇中间体还没 有被观测到. Pichler 等 ${ }^{[41]}$ 提出了 $\mathrm{CO}$ 插入机理, 认为 $\mathrm{CO}$ 加氢形成甲酰基表面物种, 进一步加氢生成桥式 氧化亚甲基物种, 然后加氢和脱水生成碳烯和甲基, 经过 $\mathrm{CO}$ 在金属-氢键、金属-烷基键中反复插入和加 氢形成 $\mathrm{C}-\mathrm{C}$ 键而完成链的增长. 目前普遍认为, 在复 杂的费托合成反应体系中可能不是受单一的反应机 理控制的, 费托合成产物的多样性及其分布特征是 几种反应机理综合作用的结果.

对于费托合成铁基催化剂, 一般认为活性相是 铁碳化物, 典型物相是 $\mathrm{Fe}_{5} \mathrm{C}_{2}$. 曹东波等课题组 ${ }^{[42 \sim 47]}$ 采用密度泛函理论方法研究了 $\mathrm{Fe}_{x} \mathrm{C}_{y}$ 相的不同晶面上 的 $\mathrm{H}_{2} 、 \mathrm{CO}$ 和合成气 $\left(\mathrm{H}_{2}+\mathrm{CO}\right)$ 吸附和解离机理, 指出 了氢助解离 $\mathrm{CO}$ 的重要性, 阐明了表面碳链增长单体 及其来源. 结合考虑碳化物机理和 $\mathrm{CO}$ 插入机理, 设 想了链引发反应生成 $\mathrm{C}_{2}$ 物种 (烃和醇)的各种可能路 径, 计算了所有 $\mathrm{C}_{1}-\mathrm{C}_{1}$ 的键合反应, 在有利于形成 $\mathrm{C}_{2}$ 中间体基础上, 进一步计算了 $\mathrm{C}_{2}-\mathrm{C}_{1}$ 的键合反应, 认
为表面中间体乙烯酮是生成 $C_{2}$ 的烃和有机含氧化合 物的关键物种, 乙烯酮加氢可以导致各种有机含氧 化合物和烃的形成，表面中间体涉及乙酰基、乙醛、 乙烷基、次乙基等. $\mathrm{C}-\mathrm{O}$ 键的断裂反应可以形成各种 烃, 如亚乙烯基 $\left(\mathrm{H}_{2} \mathrm{C}_{\mathrm{s}} \mathrm{C}\right)$ 、次乙烷基 $\left(\mathrm{H}_{3} \mathrm{C}_{\mathrm{s}} \mathrm{C}\right)$ 、乙烯基 $\left(\mathrm{H}_{2} \mathrm{C}_{\mathrm{s}} \mathrm{CH}\right)$ 、亚乙烷基 $\left(\mathrm{H}_{3} \mathrm{C}_{\mathrm{s}} \mathrm{CH}\right)$ 等. 乙烯基加氢可生成 乙烯 $\left(\mathrm{H}_{2} \mathrm{C}_{\mathrm{s}} \mathrm{CH}_{2}\right)$ 、乙烷基 $\left(\mathrm{H}_{3} \mathrm{C}_{\mathrm{s}} \mathrm{CH}_{2}\right)$ 、乙烷 $\left(\mathrm{H}_{3} \mathrm{C}_{\mathrm{s}} \mathrm{CH}_{3}\right)$ (图 1). 乙烯形成的主要反应路径是 $\mathrm{C}_{\mathrm{s}} \mathrm{H}_{2} \mathrm{CO} \rightarrow \mathrm{CC}_{\mathrm{s}} \mathrm{H}_{2} \rightarrow$ $\mathrm{CHC}_{\mathrm{s}} \mathrm{H}_{2} \rightarrow \mathrm{CH}_{2} \mathrm{C}_{\mathrm{s}} \mathrm{H}_{2}$, 乙烷形成的主要路径是 $\mathrm{C}_{\mathrm{s}} \mathrm{H}_{2} \mathrm{CO}$ $\rightarrow \mathrm{CC}_{\mathrm{s}} \mathrm{H}_{2} \rightarrow \mathrm{CC}_{\mathrm{s}} \mathrm{H}_{3} \rightarrow \mathrm{CC}_{\mathrm{s}} \mathrm{H}_{3} \rightarrow \mathrm{CHC}_{\mathrm{s}} \mathrm{H}_{3} \rightarrow \mathrm{CH}_{2} \mathrm{C}_{\mathrm{s}} \mathrm{H}_{3} \rightarrow$ $\mathrm{CH}_{3} \mathrm{C}_{\mathrm{s}} \mathrm{H}_{3}$, 乙醇形成的主要反应路径是 $\mathrm{C}_{\mathrm{s}} \mathrm{H}_{2} \mathrm{CO} \rightarrow$ $\left[\mathrm{C}_{\mathrm{s}} \mathrm{H}_{3} \mathrm{CO}\right.$ and/or $\left.\mathrm{C}_{\mathrm{s}} \mathrm{H}_{2} \mathrm{CHO}\right] \rightarrow \mathrm{C}_{\mathrm{s}} \mathrm{H}_{3} \mathrm{CHO} \rightarrow \mathrm{C}_{\mathrm{s}} \mathrm{H}_{3} \mathrm{CH}_{2} \mathrm{O} \rightarrow$ $\mathrm{C}_{\mathrm{s}} \mathrm{H}_{3} \mathrm{CH}_{2} \mathrm{OH}$ (图 2). 烃的形成比逐步加氢生成乙醇更 为有利, 乙烯酮中间物种的加氢主要产物应该是烃, 而不是有机含氧化合物, 这与费托合成产物分布中 $\mathrm{C}_{2}$ 产物选择性规律是一致的.

Lox 等 ${ }^{[48,49}$ 较早基于费托合成碳化物机理的详 细基元反应步骤建立了烷烃和烯烃生成的详细动力 学模型, 但与实际的产物分布有一定的偏差, 马文平 等 ${ }^{[50,51]}$ 在碳化物机理的基础上考虑到烯烃再吸附的 机理, 基于详细的基元反应步骤推导并结合等温积 分固定床反应的实验数据, 拟合获得了包含烷烃、烯 烃详细产物分布和水煤气变换反应产物的费托合成 动力学模型, 改进了 Lox 模型的缺陷. 随后王逸凝

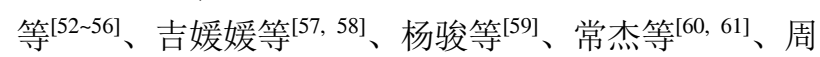
利平等 ${ }^{[62]}$ 进一步完善和改进了这一模型, 并在转篮 式无梯度反应器上获得基于详细机理的动力学模型. 滕波涛等 ${ }^{[63 ~ 68]}$ 提出了采用无梯度反应器, 利用反应 器内反应气氛、温度均一的优势, 将复杂反应动力学 解耦, 对烃、有机含氧化合物生成、水煤气变换反应 单独进行动力学建模与估算, 进而耦合获得 F-T 合成 综合模型的方法. 利用该方法, 基于碳化物机理、 $\mathrm{CO}$ 插入机理和烯烃再吸附机理, 进一步提出了一个更

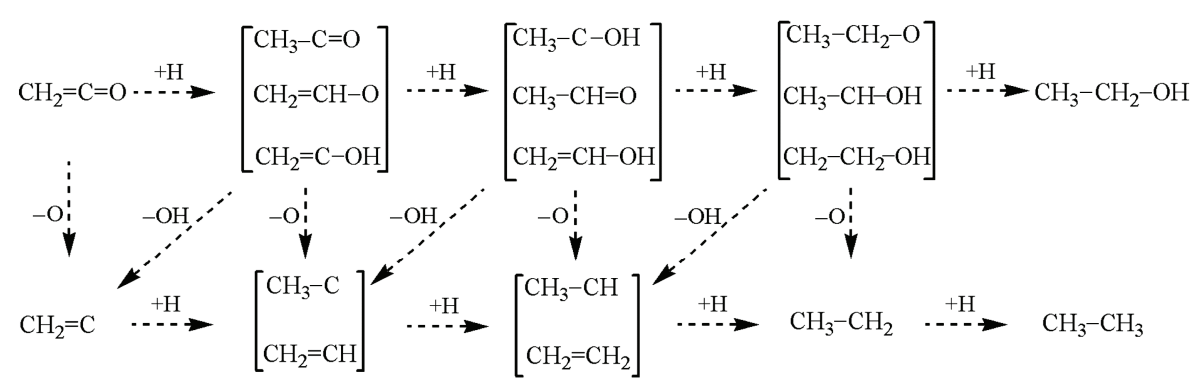

图 1 乙烷和乙醇生成的基元反应步骤

1880 


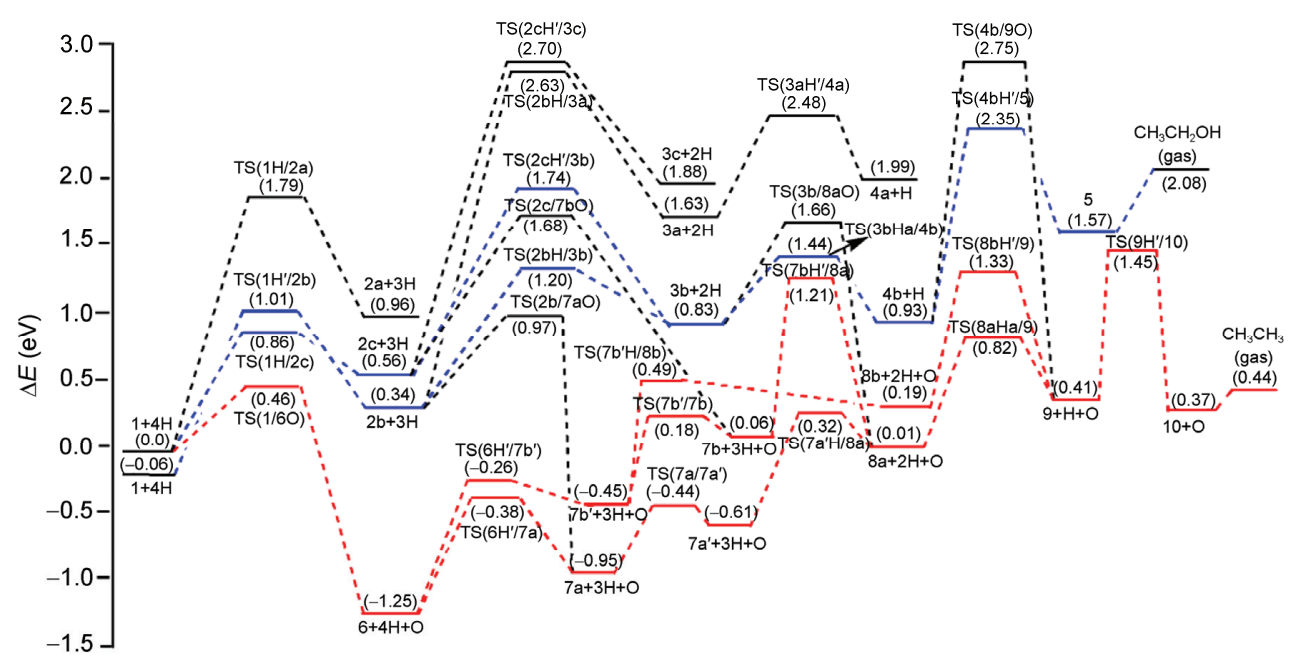

图 2 有机含氧化合物(上)和烃(下)生成的势能面 (1: $\mathrm{C}_{\mathrm{s}} \mathrm{H}_{2} \mathrm{CO} ; 2 \mathrm{a}: \mathrm{C}_{\mathrm{s}} \mathrm{H}_{2} \mathrm{COH} ; 2 \mathrm{~b}: \mathrm{C}_{\mathrm{s}} \mathrm{H}_{3} \mathrm{CO} ; 2 \mathrm{c}: \mathrm{C}_{\mathrm{s}} \mathrm{H}_{2} \mathrm{CHO} ; 3 \mathrm{a}: \mathrm{C}_{\mathrm{s}} \mathrm{H}_{3} \mathrm{COH} ; 3 \mathrm{~b}$ : $\mathrm{C}_{\mathrm{s}} \mathrm{H}_{3} \mathrm{CHO}$; $4 \mathrm{a}: \mathrm{C}_{\mathrm{s}} \mathrm{H}_{3} \mathrm{CHOH} ; 4 \mathrm{~b}: \mathrm{C}_{\mathrm{s}} \mathrm{H}_{3} \mathrm{CH}_{2} \mathrm{O} ; 5: \mathrm{C}_{\mathrm{s}} \mathrm{H}_{3} \mathrm{CH}_{2} \mathrm{OH} ; 6: \mathrm{CC}_{\mathrm{s}} \mathrm{H}_{2} ; 7 \mathrm{a}: \mathrm{CC}_{\mathrm{s}} \mathrm{H}_{3} ; 7 \mathrm{~b}: \mathrm{CHC}_{\mathrm{s}} \mathrm{H}_{2} ; 8 \mathrm{a}: \mathrm{CHC}_{\mathrm{s}} \mathrm{H}_{3} ; 8 \mathrm{~b}: \mathrm{CH}_{2} \mathrm{C}_{\mathrm{s}} \mathrm{H}_{2} ; 9$ : $\mathrm{CH}_{2} \mathrm{C}_{\mathrm{s}} \mathrm{H}_{3}, 10: \mathrm{CH}_{3} \mathrm{C}_{\mathrm{s}} \mathrm{H}_{3}$ )

详细和全面综合的费托合成基元反应机理图式(图 3), 结合水煤气变换反应的详细机理进行推导并依据转 篮式无梯度反应器上的实验数据拟合出包含烷、烯、 醇、酸生成和水煤气变换反应的费托合成统一动力学 模型(图 4). 由费托合成综合反应动力学模型计算的 合成气消耗速率、甲烷生成速率和水煤气变换反应速 率较好地与实验值吻合, 且可较好地模拟烷、烯、醇、 酸等产物的分布情况(图 5).

\section{3 费托合成铁基催化剂的结构设计和制备}

浆态床费托合成工业铁基催化剂的研发与放大 生产技术是煤间接液化工业技术开发的核心内容之 一. 反应温度的提高有利于提高费托合成过程副产 蒸汽的压力等级, 进而提高蒸汽利用效率. 但受制于 热力学和动力学的控制, 产物选择性会严重轻质化,
甲烷选择性升高; 同时催化剂会过度碳化而降低抗 磨损性能, 增加过滤负荷和难度. 因此催化剂的研发 需要解决的关键问题是：如何在较高温下获得稳定 的物相结构、高的合成气转化能力、低的甲烷和有机 含氧化物选择性、高的有效烃 $\left(\mathrm{C}_{3+}\right.$ 烃 $)$ 的选择性和良好 的抗磨损性能，同时生产过程工艺简单、绿色环保、 消耗(能耗和水耗)和制造成本低.

费托合成铁基催化剂在实际工业反应条件下， 其铁物相具有多相共存的高度复杂的特性, 涉及 $\mathrm{Fe}_{5} \mathrm{C}_{2} 、 \mathrm{Fe}_{3} \mathrm{C} 、 \mathrm{Fe}_{2.2} \mathrm{C} 、 \mathrm{Fe}_{3} \mathrm{C}_{7} 、 \alpha-\mathrm{Fe} 、 \mathrm{Fe}_{2} \mathrm{O}_{3} 、 \mathrm{Fe}_{3} \mathrm{O}_{4}$ 等的物相结构, 使得利用传统实验方法详细定义和 理解活性相上的反应过程相当困难. 在将催化剂置 于实际反应过程中的研究理念下, 采用量子力学理 论计算方法, 结合对催化剂详细结构的剖析以及对 费托合成催化机理的深入认识, 将有助于开发适于

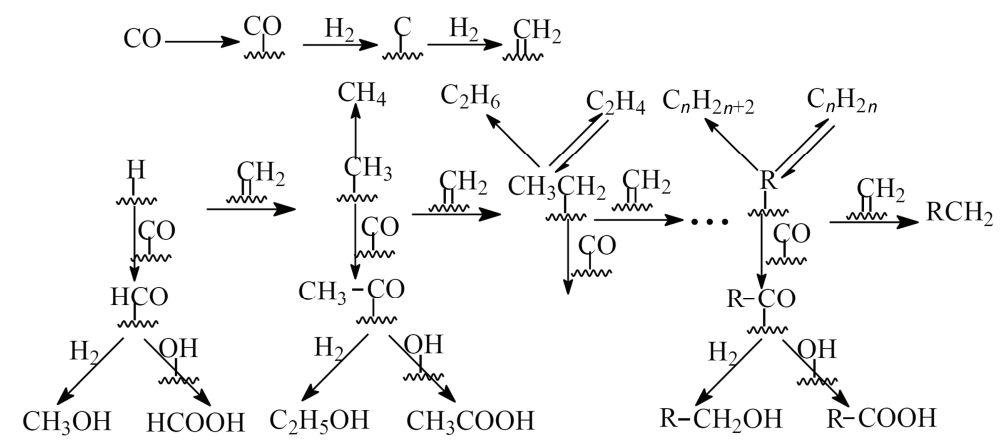

图 3 费托合成综合机理模型图式 


$$
\begin{aligned}
& R_{\mathrm{CH}_{4}}=k_{11,1}\left[\mathrm{CH}_{3} \mathrm{~S}\right][\mathrm{HS}]=k_{11,1} \alpha_{T, 1} K_{2} P_{\mathrm{H}_{2}}[\mathrm{~S}]^{2} \\
& R_{\mathrm{C}_{n} \mathrm{H}_{2 n+2}}=k_{11}\left[\mathrm{C}_{n} \mathrm{H}_{2 n+1} \mathrm{~S}\right][\mathrm{HS}]=k_{11} K_{4} P_{\mathrm{H}_{2}}[\mathrm{~S}]^{2} \prod_{i=1}^{n} \alpha_{T, i} \\
& R_{\mathrm{C}_{n} \mathrm{H}_{2 n}}=k_{12}\left[\mathrm{C}_{n} \mathrm{H}_{2 n+1} S\right]-k_{12}^{-} P_{C_{n} H_{2 n}}[\mathrm{HS}]=k_{12} \sqrt{K_{4} P_{H_{2}}}[\mathrm{~S}] \prod_{i=1}^{n} \alpha_{T, i}\left(1-\beta_{n}\right) \\
& R_{\mathrm{CH}_{3} \mathrm{OH}}=k_{9,1} K_{1} K_{4} K_{7} K_{8} P_{\mathrm{CO}} P_{\mathrm{H}_{2}}^{2}[\mathrm{~S}]^{2} \\
& R_{\mathrm{C}_{n} \mathrm{H}_{2 n+1} \mathrm{OH}}=k_{9} K_{1} K_{4} K_{7} K_{8} P_{C O} P_{\mathrm{H}_{2}}^{2} \alpha_{T}^{n-2} \alpha_{T, 1}[\mathrm{~S}]^{2} \\
& R_{\mathrm{HCOOH}}=k_{10,1} K_{1} K_{7} P_{\mathrm{CO}} P_{\mathrm{H}_{2} \mathrm{O}}[\mathrm{S}]^{2} / K_{6} \\
& R_{\mathrm{C}_{n} \mathrm{H}_{2 n-1} \mathrm{OOH}}=k_{10} K_{1} K_{7} P_{\mathrm{CO}} P_{\mathrm{H}_{2} \mathrm{O}} \alpha_{T}^{n-2} \alpha_{T, 1}[\mathrm{~S}]^{2} / K_{6} \\
& R_{\mathrm{CO}_{2}}=\frac{k_{W 2} K_{W 1} K_{W 3} P_{\mathrm{CO}} P_{\mathrm{H}_{2} \mathrm{O}}-k_{W-2} P_{\mathrm{H}_{2}} P_{\mathrm{CO}_{2}}}{\sqrt{K_{W 3} P_{\mathrm{H}_{2}}}+P_{\mathrm{H}_{2}}+K_{W 1} P_{\mathrm{CO}} P_{\mathrm{H}_{2} \mathrm{O}} / P_{\mathrm{H}_{2}}}
\end{aligned}
$$

图 4 包含烷、烯、醇、酸生成和水煤气变换反应的费托合成统一动力学模型

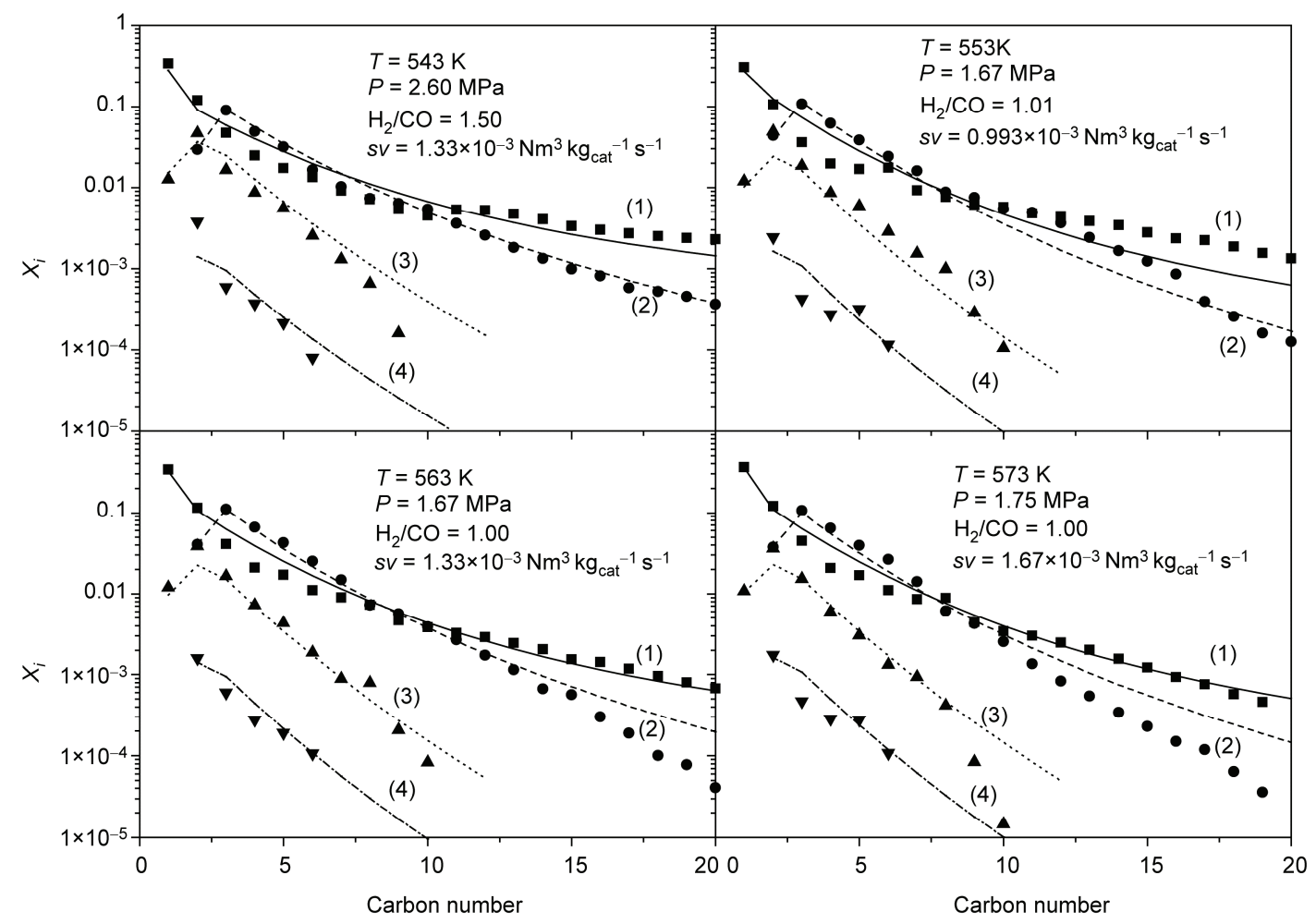

图 5 烷(1)、烯(2)、醇(3)、酸(4)产物分布的实验值与计算模拟值的对比(图中符号为实验值, 线为计算值)

高温浆态床操作的铁基催化剂.

霍春芳等 ${ }^{[69]}$ 应用密度泛函理论(DFT)研究了一 系列铁碳化物的不同物相、不同晶面上的 F-T 反应机 理及 $\mathrm{H}_{2}$ 和 $\mathrm{CO}$ 在铁碳化物晶面的吸附与烃成键行为,
发现在 $\mathrm{Fe}_{5} \mathrm{C}_{2}(010)$ 和 $\mathrm{Fe}_{2} \mathrm{C}(011)$ 表面容易形成甲烷， 而 $\mathrm{Fe}_{4} \mathrm{C}(100)$ 和 $\mathrm{Fe}_{3} \mathrm{C}(001)$ 表面对甲烷化反应活性较低, 确定了最可能的铁基催化剂的活性相及晶面结构取 向，在理论上发现了甲烷形成的反应能与有效势垒 
分别与铁碳化物表面 $\mathrm{C}$ 原子的电荷及表面的 $\mathrm{d}$ 带中 心能量呈线性相关(图 6), 这种构效关系的建立, 可 通过分析各种铁碳化物表面性质来预测催化剂的反 应性和烃产物选择性. $\mathrm{K}$ 助剂是费托合成铁基催化剂 不可或缺的关键组分, 长期以来国际上对 $\mathrm{K}$ 助剂作 用机理的认识一直处于 “黑箱” 状态, 一般认为其作 用是电子效应. 霍春芳等课题组 ${ }^{[70 ~ 72]}$ 基于密度泛函 理论和实验研究结果发现, $\mathrm{K}$ 助剂的作用还在于调变 铁微晶的暴露晶面, 从而控制产物分布. $\mathrm{K}$ 助剂 $\left(\mathrm{K}_{2} \mathrm{O}\right)$ 对 Fe(110)、(100)、(111)、(211)、(210)、(321)和(310) 面具有不同程度的稳定化作用. 通过调变 $\mathrm{K}$ 含量, 可 以改变铁微晶在不同晶向的相对生长速度, 使得铁 微晶暴露更多的高活性晶面. 对于不含 $\mathrm{K}$ 助剂的催 化剂, 主要暴露 $\mathrm{Fe}(110)$ 面; $\mathrm{K}$ 的添加导致(110)和(100) 面占铁微晶总表面的比例减少, 高活性晶面(211)和 (310)的比例增加(图 7), 这一结果突破了对 K 助剂催 化作用的传统认识. 上述结果对研制高活性、高选择 性、晶面取向可控的费托合成催化剂起到了重要的理 论指导作用.

在铁基催化剂制备、结构和反应性能研究方面,

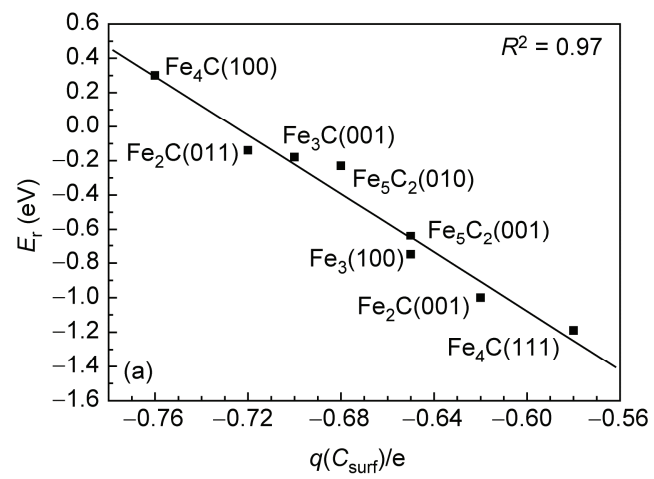

杨勇等 ${ }^{[73 ~ 84]}$ 通过调整助剂 $\mathrm{K} 、 \mathrm{Mn} 、 \mathrm{Cu} 、 \mathrm{Ca} 、 \mathrm{Mg}$ 、 $\mathrm{Ni}$ 与黏结剂 $\mathrm{SiO}_{2} 、 \mathrm{Al}_{2} \mathrm{O}_{3}$ 及制备参数等, 系统地考察 了铁基催化剂的制备化学规律, 调控催化剂体相和 表面结构(图 8), 结合活性相铁碳化物上的合成气反 应生成烃的链增长机理, 掌握了制约烃产物分布的 基本规律和催化剂的磨损机理, 找到了催化剂预处 理还原与实际反应过程中的物相变化、反应活性与产 物选择性、磨损性能间的平衡协调关系(图 9), 可控 制催化剂定向还原至合适碳化铁含量与合理的有效 晶面取向范围 ${ }^{[85 ~ 89]}$, 显著减少了催化剂的物理膨胀 应力和化学腐蚀磨损(图 10), 保障了催化剂在有利于 烃链增长(柴油选择性高)和高活性(柴油产率高) 的 活性相下进行费托反应, 解决了制约催化剂稳定运 行的磨损问题.

在催化剂制备生产技术上，突破了传统的合成 催化剂的制备思路, 以廉价的铁为起始原料, 溶化铁 产生的 $\mathrm{NO}_{x}$ 采用低温加压水吸收技术生产稀硝酸并 回用, $\mathrm{NO}_{x}$ 排放 $<50 \mathrm{ppm}$, 沉淀后使用压滤、精滤与套 洗集成工艺, 有效降低了水耗(<20 吨水/吨催化剂), 滤 液 $\mathrm{Fe}$ 含量 $<3 \mathrm{ppm}$, 实现了滤液中无机盐的回收;

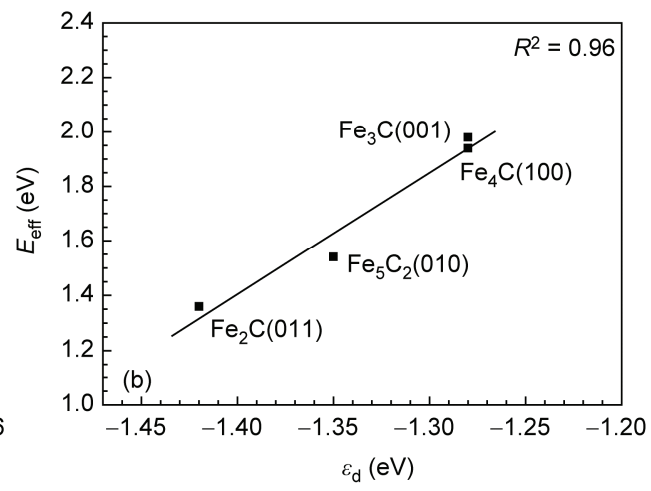

图 6 (a) 形成甲烷的反应能 $\left(E_{\mathrm{r}}\right)$ 与表面 $\mathrm{C}$ 原子电荷 $(q)$ 的关系; (b) 甲烷形成的表面势垒 $\left(E_{\mathrm{eff}}\right)$ 与表面 $\mathrm{C}$ 原子 $\mathrm{d}$ 带中心能量 $\left(\varepsilon_{\mathrm{d}}\right)$ 的关系

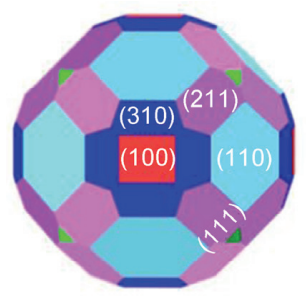

(a) $\mathrm{K} / \mathrm{Fe}=0$

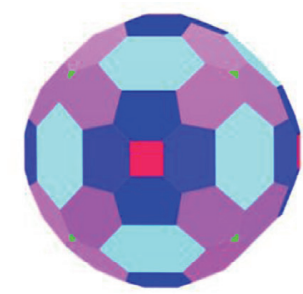

(b) $\mathrm{K} / \mathrm{Fe}=1 / 48$

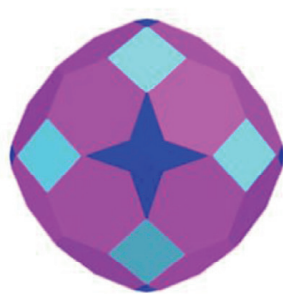

(c) $\mathrm{K} / \mathrm{Fe}=1 / 12$

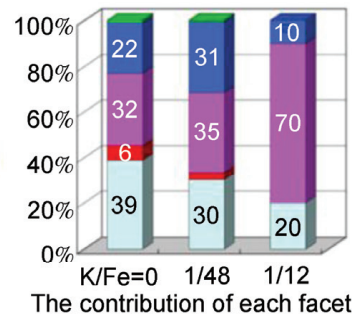

The contribution of each facet

图 7 晶面取向随铁基催化剂助剂 $\mathrm{K}$ 含量的变化 


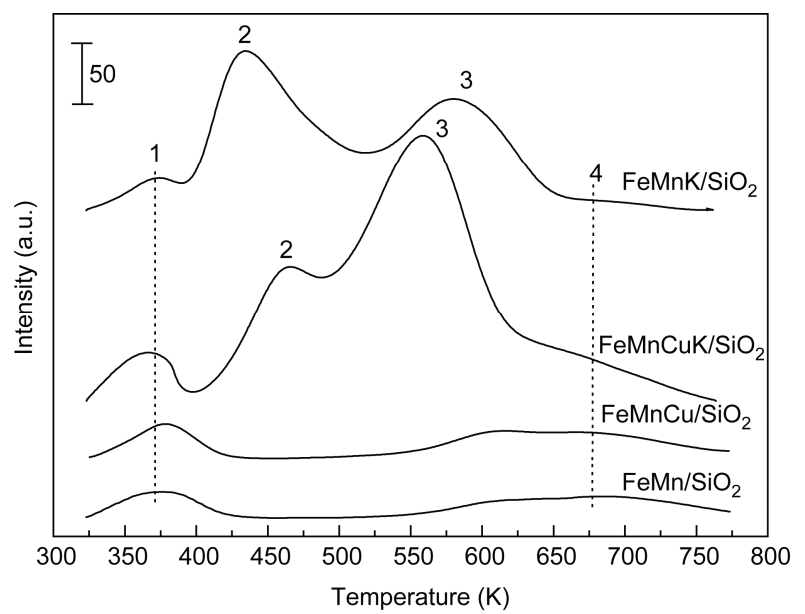

图 8 铁基催化剂表面碱性的调控: 催化剂表面的 $\mathrm{CO}_{2}-$ TPD 谱图

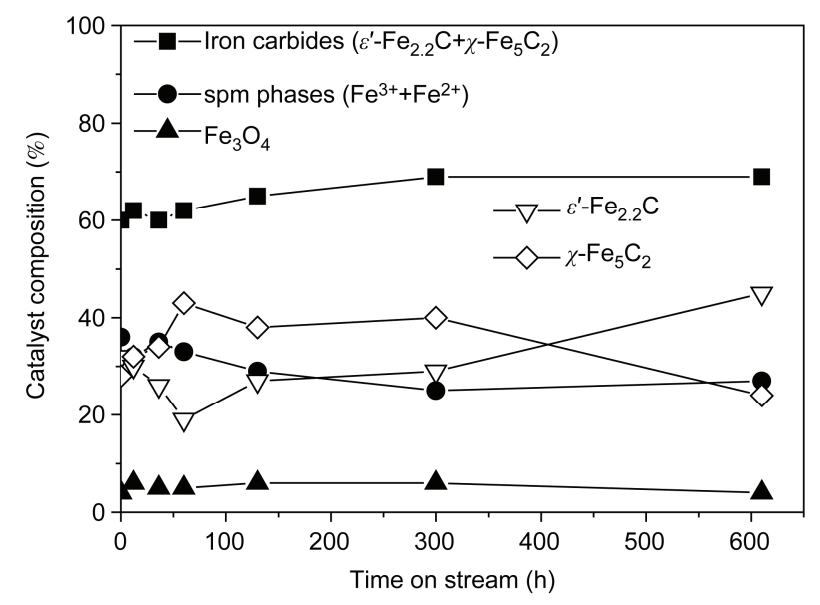

图 9 铁基催化剂在费托合成反应过程中的物相变化

采用发明的催化剂成型技术及黏结剂添加技术制得 高抗磨损的微米级球状催化剂(30 200 $\mu \mathrm{m}$, 图 10),

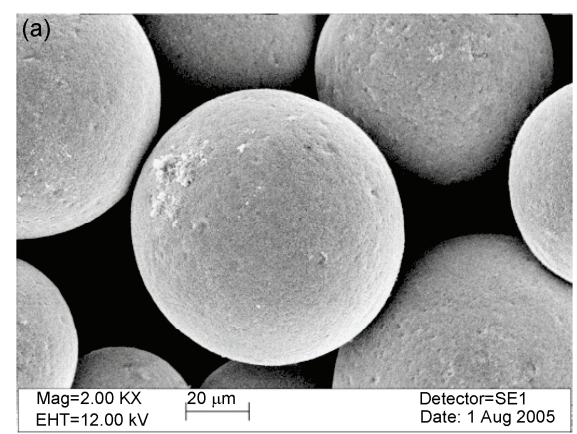

成品率 > 99\%, 实现了高温浆态床费托合成工业铁 基催化剂的绿色工艺生产, 同时铁基催化剂的生产 成本得到大幅度降低 ${ }^{[89 ~ 93]}$.

\section{4 浆态床合成反应器设计与控制}

在浆态床反应器技术方面, 国外在关键的内构 件和蜡催化剂分离技术方面一直很保密, 真正工业 应用的技术在公开的国际专利中难以获取. 李永旺 等 ${ }^{[92 ~ 98]}$ 基于费托合成详细机理动力学, 通过涉及产 物在床层分布的传质传热的多级两相 CSTR 稳态等温 模型, 结合 CFD 流体力学对浆态床反应器内部流场分 析和冷态模拟验证 $(\varphi 1200 \mathrm{~mm})$, 建立了浆态床反应器 模型, 自主设计制造了内径 5.3 5.8 m, 高 $48 \mathrm{~m}$ 、单 台产能达 16 20 万吨/年的大型高温浆态床反应器(图 11).

设计的浆态床合成反应器 ${ }^{[94,95,99]}$ 具有如下优势: (1) 采用器内在线过滤方案, 发明了内外对置、反吹 和精滤集成的蜡-催化剂分离器技术, 通过特殊设计 的内部过滤构件和外部控制、操作系统, 在线实现了 反应器内液相产物和催化剂的高效分离, 催化剂一 次分离结果为排出蜡液中的固体含量 $<5 \mathrm{ppm}$, 精滤 后固体杂质含量 $<1 \mathrm{ppm}$. (2) 气体分布器采用反向 密布气孔的雉盘式结构, 保障合成气在主体流场中 的均匀分布, 有效抑制了气泡的聚集与返混. (3) 塔 顶消泡装置采用多层间插式导流槽结构, 解决了高 温操作下可能产生的液泛或冲顶; 优化布局的换热 器系统采用列管盘式分层强制换热结构, 高效移出反 应热(蒸汽压力 $2.0 \sim 3.0 \mathrm{MPa}$ ), 解决了高温操作下的费 托合成强放热及热量高效回收难题. (4) 设计了独特的 体外还原反应器和输送添加单元, 在反应运行过程

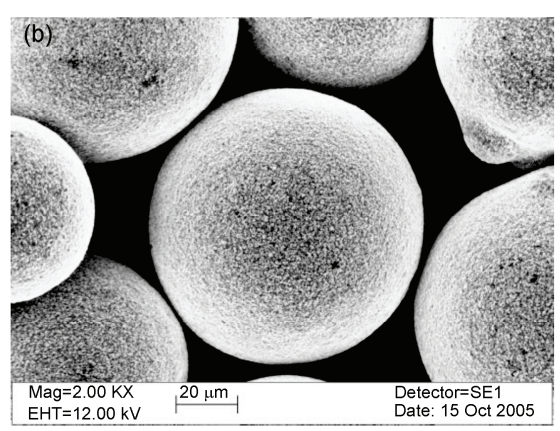

图 10 研制的浆态床合成工业铁基催化剂. (a) 制备的铁基催化剂形貌; (b) 铁基催化剂运行 $1240 \mathrm{~h}$ 后的形貌 


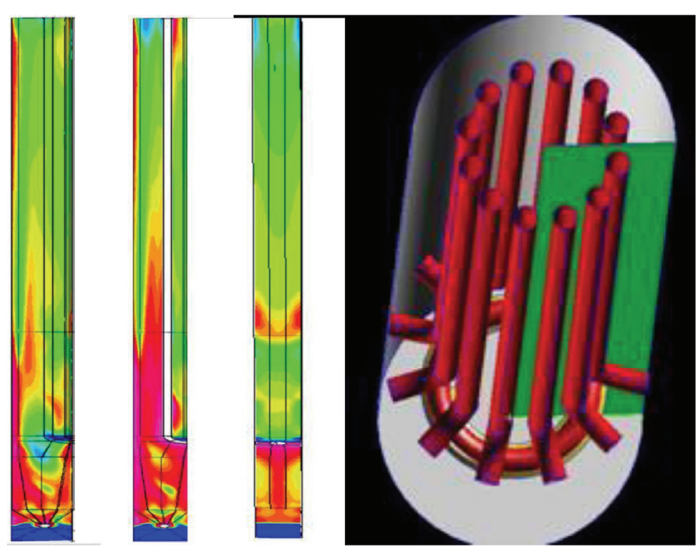

图 11 浆态床合成反应器内设置内构件及其流体力学模拟

中就可进行催化剂预还原和连续向反应器内添加催 化剂, 同时反应器上某处内构件又可将破损的催化剂 适时连续移出, 使浆态床反应器可实现连续长周期稳 定运行.

设计的大型浆态床反应器成功实施了现场组焊 和吊装, 伊泰和潞安两个 16 万吨/年合成油示范厂运 行结果证明该反应器运行平稳, 轴向温差 $<1{ }^{\circ} \mathrm{C}$, 径 向温差 $<3^{\circ} \mathrm{C}$, 在换热、物料分布及产品分离等方面 体现出优异的工程性能. 进一步形成了单台产能 50 80 万吨/年、内径 $10 \mathrm{~m}$ 级的大型浆态床反应器设计 与制造方案.

\section{5 油品加工}

煤炭间接液化合成出的粗产品主要为长链烷烯 烃, 具有无硫、无氮、低芳烃和环烷烃的特点, 其油 品加工技术与原油加工相比, 还存在着一定的差异 性 ${ }^{[100]}$. 李永旺等 ${ }^{[101 ~ 104]}$ 研发出 $\mathrm{Ni}$ 基非硫化态加氢精 制催化剂及油品加氢工艺, 该催化剂在加氢过程中 无需补硫, 能够有效地将烯烃加氢饱和转化为相应 的烷烃, 同时可以有效地脱除油品中的有机含氧化 合物, 生产出直馏柴油和石脑油产品. 该油品加氢工 艺及其催化剂, 与高温浆态床费托合成工艺相结合, 在 16 万吨/年合成油示范厂中生产出的高品质柴油, 其 $\mathrm{S}$ 含量 $<0.5 \mathrm{ppm}$, 多环芳烃 $<1 \%$, 十六烷值 $>$ 74 , 整个煤制油工艺过程产品分布为柴油占 63\% 67\%, 石脑油占 $25 \%$ 28\%, LPG 占 10\%. 生产出 的柴油经台架试验表明, 与市场 0 号柴油相比, 各项 尾气排放指标显著下降 ${ }^{[105]}$, 同时使用合成柴油可节 约 8\% 12\%的油耗，达到了欧 V 柴油的标准.
针对费托合成蜡的加工，许丽恒等 ${ }^{[106,107]}$ 通过对 石油加工所用 Stangeland 模型的改进, 按结构族组成 和碳数范围将原料蜡和加氢裂化产物划分为 9 个虚 拟组分, 建立了更为准确的蜡加氢裂解的集总动力 学模型, 揭示了工艺参数对加氢裂化产物选择性和 收率的影响规律, 为费托合成蜡加氢裂解生产柴油 的产品调控和反应器设计提供了理论依据.

在目前实施的百万吨级商业装置上, 为进一步 提高过程的经济性, 丰富产品结构, 将增加低碳烯烃 芳构化技术和尾气流向变换催化燃烧反应器技术的 应用, 生产出优质的高辛烷值汽油调和组分.

\section{6 工艺系统集成}

基于能效和过程经济性原则, 采用以费托合成 详细机理动力学模型和浆态床反应器模型为核心编 写的煤制油全流程工艺模拟软件, 对煤气化(气化炉) 水煤浆气化、碎煤加压气化、Shell 气化等)-气体净化费托合成(高/低温浆态床、固定床)-油品加工-化学品发电等多种组合工艺方案进行了局部和全流程模拟 计算与优化, 形成了示范厂(16 20 万吨/年)和百万吨 级商业厂 $\left(100 \sim 540\right.$ 万吨/年)系统集成方案 ${ }^{[108 ~ 112]}$.

示范厂运行中副产的 2.0 3.0 MPa 高品位蒸气伊 泰和潞安示范厂用于发电和全厂热量平衡, 验证了 高温浆态床合成工艺概念，系统集成优化后：(1) 高 品位蒸汽的合理利用提高了总体能效约 2 个百分点; （2）性能优异的催化剂使得甲烷选择性降低到 3\%以 下, 减少了尾气甲烷转化的负荷, 提高总能效率约 1 个百分点; (3) 各单元过程的全局优化集成, 提高了 总能效率约 1 个百分点. 由此, 使得整体煤制油效率 由低温浆态床工艺的 37\% 38\%提高到示范厂高温浆 态床工艺的 40\% 42\%. 伊泰示范厂考核表明: 合成 气总转化率为 $91 \%$ \% $2 \%$, 吨催化剂产油能力 1500 1800 吨, 全系统负荷达到 100\% 120\%, 总煤耗 3.48 吨标煤/吨油品, 系统能量转化效率达 $40.53 \%$.

目前针对不同地区煤种气化特性、产品需求及经 济性考虑, 产品方案由示范厂产品相对单一(柴油-石 脑油-LPG)向商业厂产品多元化方向发展(柴油/汽油/ 航煤-溶剂油-费托蜡-LPG-精细化学品); 基于示范厂 取得的工程数据, 通过优化模拟和系统集成正在建 设百万吨级商业厂(100 540 万吨/年). 采用节水、减 排技术(空冷与密闭循环技术、未来可能与 $\mathrm{CO}_{2}$ 封存 或资源化过程对接), 形成了多套商业厂工艺流程方 
案, 设计了单系列 50 80 万吨/年、直径 $10 \mathrm{~m}$ 级的大 型反应器, 优化后的百万吨以上规模商业厂吨催化 剂产油能力将提高至 2000 吨以上, 系统能效将提升 至 44\% 47\%.

\section{4 工业示范和产业化推广}

\section{1 合成油示范装置}

2008 年在安徽建成投产了 1500 吨/年合成油催 化剂厂, 实现了批量催化剂工业生产. 2009 年建成内 蒙古伊泰 16 万吨/年(图 12)、山西潞安 16 万吨/年和 鄂尔多斯神华 18 万吨/年的三个煤炭间接液化示范厂, 其中伊泰和潞安示范厂实现了“安稳长满优”的工业化 生产, 合成气总转化率为 $91 \%$ 92\%, 催化剂产油能力 达到 1500 1800 吨油品/吨催化剂, 费托合成中甲烷选 择性小于 $3.0 \%, \mathrm{C}_{5}$ 选择性为 $90 \%$ $94 \%, \mathrm{CO}_{2}$ 选择性为 13\% 18\%, 系统能量转化效率达到 $40.53 \%$, 合成柴油

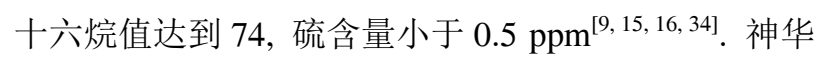
集团 18 万吨/年煤炭间接液化示范厂 2010 年进行了 两次开车验证试验, 2011 年神华宁煤 400 万吨/年煤 制油项目决定采用中科技术, 迫使南非 Sasol 合成油 技术退出中国市场 ${ }^{[18]}$. 这充分证明了我国已掌握了 可靠先进的煤炭间接液化工业技术，其中在系统能 效、催化剂产能与活性、产品选择性等关键核心技术 指标上优于国际煤炭间接液化技术水平. 表 1 为按国
外合成工艺推算的数据和自主合成油技术性能指标 的对比结果.

\section{2 百万吨级合成油商业厂建设}

以中科合成油技术有限公司为代表的我国自主 研发的煤制油技术经过“十一五”示范厂运行成功后， 在“十二五”期间已经启动了多个百万吨级煤制油商 业项目, 主要包括: 神华宁煤 400 万吨/年(图 13), 山 西潞安 180 万吨/年(一期 100 万吨/年), 新疆伊犁 540 万吨/年(一期 100 万吨/年), 新疆甘泉堡 540 万吨/年 (一期 200 万吨/年), 内蒙古准格尔 400 万吨/年(一期 200 万吨/年), 内蒙古杭锦旗 400 万吨/年(一期 120 万 吨/年), 内蒙古锡林郭勒 100 万吨/年, 贵州 500 万吨/ 年(一期 120 万吨/年). 同时在内蒙古大路煤化工园区 配套启动建设 1.2 万吨/年合成油催化剂厂. 此外, 采

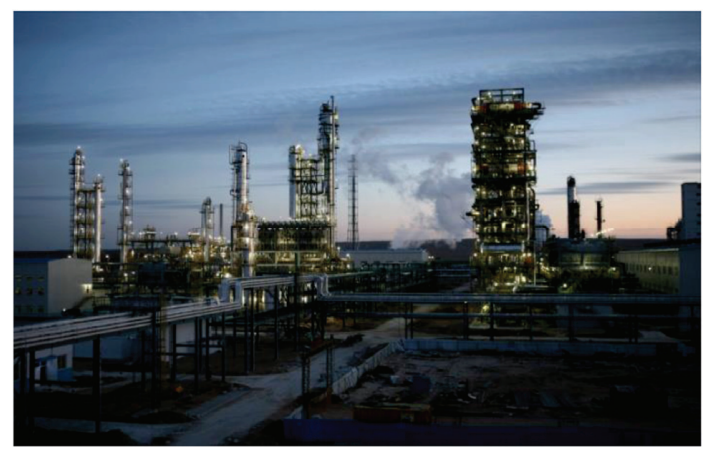

图 12 内蒙古伊泰 16 万吨/年合成油示范装置

表 1 中科合成油技术与国外合成油技术的工艺指标对比 ${ }^{[9,15,16,20 ~ 22, ~ 24, ~ 34] ~}$

\begin{tabular}{|c|c|c|c|c|c|}
\hline \multirow{2}{*}{ 工艺技术名称 } & \multirow{2}{*}{$\begin{array}{c}\text { Shell 低温固定 } \\
\text { 床工艺 }\end{array}$} & \multirow{2}{*}{$\begin{array}{c}\text { Sasol 高温流 } \\
\text { 化床工艺 }\end{array}$} & \multirow{2}{*}{$\begin{array}{c}\text { Sasol 低温 } \\
\text { 浆态床工艺 }\end{array}$} & \multicolumn{2}{|c|}{ 中科高温浆态床工艺 } \\
\hline & & & & 示范厂 & 大规模工艺计算 \\
\hline \multicolumn{6}{|l|}{ 工艺参数 } \\
\hline 催化剂 & 钴 & 铁 & 铁/钴 & \multicolumn{2}{|c|}{ 铁 } \\
\hline 反应器 & 固定床 & 流化床 & 浆态床 & \multicolumn{2}{|c|}{ 浆态床 } \\
\hline 操作温度 $\left({ }^{\circ} \mathrm{C}\right)$ & $190 \sim 220$ & $300 \sim 340$ & $200 \sim 250$ & \multicolumn{2}{|c|}{$260 \sim 290$} \\
\hline 副产蒸汽压力(MPa) & $0.5 \sim 0.8$ & $3.5 \sim 5.0$ & $0.5 \sim 0.8$ & \multicolumn{2}{|c|}{$2.5 \sim 3.0$} \\
\hline \multicolumn{6}{|l|}{ 催化剂水平 } \\
\hline 甲烷选择性(wt\%) & $5 \sim 7$ & $10 \sim 12$ & $5 \sim 6 / 8 \sim 10$ & $2 \sim 3$ & $<3$ \\
\hline 时空产率 $\left(\mathrm{g} \mathrm{C}_{3^{+}} \mathrm{HC} / \mathrm{gcat}, \mathrm{h}\right)$ & $0.20 \sim 0.25$ & 0.30 & $0.20 \sim 0.30$ & $1.0 \sim 1.5$ & $>1.0$ \\
\hline 产能(吨油/吨催化剂) & $1000 \sim 1500$ & $200 \sim 250$ & $250 / 1000$ & \multicolumn{2}{|c|}{$1500 \sim 1800$} \\
\hline $\begin{array}{c}\text { 单位油品催化剂成本 } \\
\text { (元/吨油品) }\end{array}$ & $>300$ & $>300$ & $>300$ & \multicolumn{2}{|c|}{$<100$} \\
\hline \multicolumn{6}{|l|}{ 整体工艺指标 } \\
\hline 产品加工催化剂体系 & 硫化态 & 硫化态 & 硫化态 & \multicolumn{2}{|c|}{ 非硫化态 } \\
\hline 产品中硫含量(ppm) & $<5$ & $<5$ & $<5$ & \multicolumn{2}{|c|}{$<0.5$} \\
\hline 合成气消耗 $\left(\mathrm{Nm}^{3} /\right.$ 吨油 $)$ & $5800 \sim 5900$ & 6000 & 5700 & 5400 & 5300 \\
\hline 过程总能量转换效率(\%) & $37 \sim 38$ & $38 \sim 39$ & $37 \sim 38$ & $40 \sim 42$ & $44 \sim 47$ \\
\hline
\end{tabular}

1886 


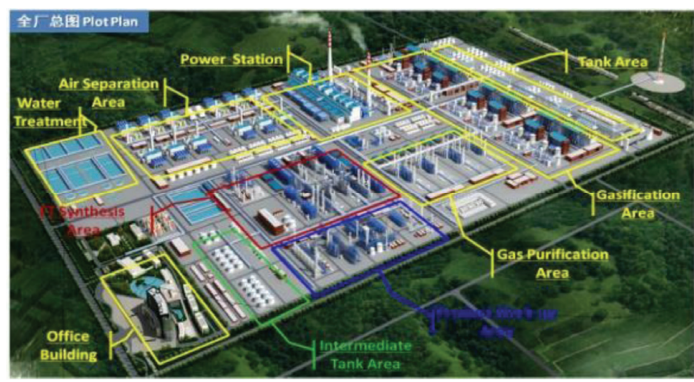

图 13 建设中的神华宁煤 400 万吨/年煤炭间接液化商业厂

用上海充矿能源科技研发有限公司开发的低温浆态 床合成技术正在陕西榆林建设一套 100 万吨/年煤制 油装置 ${ }^{[35]}$. 预计到 2016 年我国煤炭间接液化产业将 形成产能约 1600 万吨/年, 2020 年形成产能约 3100 万吨/年, 届时煤制油产量将占当年石油进口量的 $7.7 \%^{[113 \sim 115]}$.

\section{5 展望}

近 15 年来, 我国煤炭间接液化工业化技术开发 从实验室小试、工业中试、工业示范到目前正在实施 建设的百万吨级装置, 在关键的费托合成反应机理、 催化剂研制、反应动力学、反应器设计、系统工艺集 成等理论与工程技术研究方面均取得了重大突破, 自主研发和掌握了先进的高温浆态床合成油成套工 艺和工业催化剂技术, 并在 16 万吨/年合成油示范装 置上获得验证, 多项技术指标已经超过了国外同类 技术水平. 以自主合成油技术为支撑, 目前正在实施 建设多个百万吨级商业装置.

2012 年由国家发改委、国家能源局编制的《煤 炭深加工示范项目规划》以及《煤炭深加工产业发展 政策》出台实施，在“十一五”期间我国自主的煤炭间 接液化技术获得成功示范基础上, “十二五” 期间示范 技术将推进到 100 万吨级以上的重大示范项目级别 上, 同时对煤炭间接液化的能源转化效率、煤耗、水 耗、技术装备水平提出了更高、更为严苛的要求 ${ }^{[113]}$, 未来煤炭间接液化技术将进入科学有序、适度规模、 技术高度集成与提升的发展阶段, 为此, 提出以下研

\section{究对策和建议.}

（1）强化煤炭间接液化过程放大中遇到的工程 技术基础研究, 集中力量解决我国煤制油大型装备 设计、制造与安装的关键技术问题, 譬如合成反应器 由内径 $5 \mathrm{~m}$ 级提升到 $10 \mathrm{~m}$ 级、单台产能由 16 20 万 吨/年提升到 50 80 万吨/年中的工程问题, 大型煤气 化炉进一步提高气化效率和煤种适用性问题等, 全 面提升我国煤制油领域大型装备的机加工技术水平, 有效降低煤炭间接液化装置的投资规模.

（2）在单元过程技术进一步优化创新的基础上， 加强整体过程的系统集成优化工艺模拟与仿真研究, 确保在百万吨级煤间接液化装置上能源转化效率达到 $42 \%$ 以上, 单位产品综合能耗低于 4 吨(标煤)/吨油.

(3) 加强费托合成催化剂和油品加工催化剂的 基础研究, 从反应机理和活性相结构上寻求理论突 破, 指导研制活性更高、油品选择性更优、寿命更长 的工业催化剂, 降低单位催化剂油品成本, 实现催化 剂的升级换代.

(4) 开发多样性的油品加工和化学品加工新工 艺, 进一步优化产品结构, 逐步实现柴油、汽油、航 煤、溶剂油、基础润滑油、特种燃料、高附加值化学 品等多种产品生产, 满足大都市区域对无硫无氮低 芳的高品质油品的需求.

(5) 选择具备合适容量水源的地区建设煤制油 装置, 特别关注煤制油过程中的污水处理和水循环 回用技术的攻关, 采用最先进的空冷和密闭循环技 术, 控制水耗在 6 吨/吨油品以下, 实现水质达标和零 排放 ${ }^{[116]}$.

（6）积极应对煤制油过程中的碳排放问题，通过 提高煤制油装置的整体能量利用效率来实现 $\mathrm{CO}_{2}$ 相 对减排 5\% 8\%, 在煤制油装置脱碳单元可释放出纯 度 $99 \%$ 以上的 $\mathrm{CO}_{2}$, 可与未来 $\mathrm{CO}_{2}$ 捕集和封存 $(\mathrm{CCS})$ 工程对接, 实现 $\mathrm{CO}_{2}$ 绝对减排 $60 \%$ 以上, 此外也可参 与汇碳造林工程来承担社会责任 ${ }^{[17]}$.

（7）针对我国拥有难以利用的大量的低热值褐 煤资源, 积极开发煤炭分级液化技术, 实现褐煤的梯 级有效利用, 形成新一代煤制油技术 ${ }^{[18]}$. 


\section{参考文献}

1 中国石油集团经济技术研究院《2013 年国内外油气行业发展报告》课题组. 2013 年国内外油气行业发展概述及 2014 年展望. 国际 石油经济, 2014, 1-2: 30-39

2 全国煤化工信息站. 中国能源“十一五”成就、“十二五”发展规划及现状(上). 煤化工, 2013, 41: 1-4

3 焦建玲, 尤志文, 朱俊红. 我国原油进口来源改进策略分析. 中国能源, 2012, 34: 31-34

4 BP stats. BP statistical review of world energy. In: Technical Report. London: BP Amoco, 2014

5 国土资源部《全国煤炭资源潜力评价》报告. 中国煤炭地质新闻网, http://www.zmdxw.com/xinwenfabu/newshow.asp?news_id=1228, 2014 年 1 月 1 日

6 我国煤炭资源可持续可采能力概念分析. 中国行业研究网, http://www.chinairn.com/news/20130806/102805440.html, 2013-08-06

7 全国煤化工信息站. 2013 年煤炭市场数据. 煤化工, 2014, 42: 21

8 国土资源部. 全国油气资源动态评价(2012). 中国国土资源报, 2014

9 李永旺, 杨勇, 相宏伟. 煤炭间接液化产业化新进展. 见: 中国科学院, 主编. 2014 高技术发展报告. 北京: 科学出版社, 2014. $222-228$

10 Hao X, Dong GQ, Yang Y, Xu YY, Li YW. Coal to liquid (CTL): commercialization prospects in China. Chem Eng Tech, 2007, 30: $1157-1165$

11 相宏伟, 唐宏青, 李永旺. 煤化工工艺技术评述与展望IV. 煤间接液化技术. 燃料化学学报, 2001, 29: 290-298

12 Schulz H. Short history and present trends of Fischer-Tropsch synthesis. Appl Catal A: Gen, 1999, 186: 3-12

13 高晋生, 张德祥. 煤液化技术. 北京: 化学工业出版社, 2005

14 赵宗燠. 我国合成石油工程开创的回忆. 见: 武衡, 主编. 东北区科学技术发展史资料(10). 解放战争时期和建国初期. 石油工业. 北京: 中国学术出版社, 1989. 327

15 Li YW, Xu J, Yang Y. Diesel from Syngas. New York: Wiley, 2009

16 Li YW, de Klerk A. Industrial Case Studies. New York: Wiley, 2013

17 Liu ZY, Shi SD, Li YW. Coal liquefaction technologies-development in China and challenges in chemical reaction engineering. Chem Eng Sci, 2010, 65: 12-17

18 穆阳. 沙索的宁煤滑铁卢. 中国煤化工, 2011, 1: 54-57

19 Fischer F, Tropsch H. Synthesis of petroleum at atmospheric pressure from gasification products of coal. Brennstoff-Chemie, 1926, 7: $97-104$

20 Dry ME. High quality diesel via the Fischer-Tropsch process-a review. J Chem Tech Biotech, 2002, 77: 43-50

21 Dry ME. The Fischer-Tropsch process: 1950-2000. Catal Today, 2001, 71: 227-241

22 Dry ME. Fischer-Tropsch synthesis-industrial. In: Encyclopedia of Catalysis. New York: Wiley, 2010. 1-3

23 Sasol reports progress on US gas-to-liquids plant. Focus on Catal, 2014, 3: 6-7

24 van Wechem VMH, Senden MMG. Conversion of natural gas to transportation fuels via the Shell middle distillate synthesis process(SMDS). Stud Surf Sci Catal, 1994, 81: 43-71

25 Carlsson L, Fabricius N. From bintulu shell MDS to pearl GTL in qatar. In: Gastech 2005. Bilbao, 2005. 1-10

26 Shell international limited. Pearl GTL-an overview. http://www.shell.com/global/aboutshell/ major-projects-2/pearl/overview.html, 2014-07-29

27 相宏伟, 钟炳. 天然气制取液体燃料工艺技术研究进展. 化学进展, 1999, 11: 386-394

28 陈家否. 中国煤液化技术兴衰历程初析. 中国科技史杂志, 2013, 34: 199-212

29 周敬来, 张志新，张碧江. 煤基合成液体燃料的 MFT 工艺技术. 1999, 27: 58-64

30 张志新, 胡靖文, 唐曙光, 边定国, 孔令媛，陈冬花，李毅. 煤制合成油工业单管试验. 天然气化工, 1997, 24: 24-28

31 孙予罕, 李永旺. 煤基浆态床合成油品的工业化. 中国科学院院刊, 2002, 2: 100-103

32 Bai L, Xiang HW, Li YW, Han YZ. Slurry phase Fischer-Tropsch synthesis over manganese-promoted iron ultrafine particle catalyst. Fuel, 2002, 81: 1577-1581

33 白亮, 邓蜀平，董根全，曹立仁，相宏伟，李永旺. 煤间接液化技术开发现状及工业前景. 化工进展, 2003, 22: 441-446

34 唐宏青. 煤制油 F-T 前进的步伐. 中氮肥, 2014, 1: 6-9

35 孙启文. 煤炭间接液化. 北京: 化学工业出版社, 2012

36 Steynberg A, Dry M. Fischer-Tropsch Technology. Amsterdam: Elsevier, 2004

37 Lee WH, Bartholomew CH. Multiple reaction states in CO hydrogenation on alumina-supported cobalt catalysts. J Catal, 1989, 120: $256-171$ 
38 Anderson RB. The Fischer-Tropsch Synthesis. Florida: Academic Press Inc, 1984

39 Storch HH, Goulombic N, Anderson RB. The Fischer-Tropsch and Related Synthesis, Including a Summary of Theoretical and Applied Contact Catalysis. New York: Wiley, 1951

40 Eidus YT. The mechanism of the Fischer-Tropsch reaction and the initial hydropolymerisation of alkenes from radiochemical and kinetic data. Russ Chem Rev, 1967, 36: 338-348

41 Pichler H, Shulz H. Neuere erkenntnisse auf dem gebiet der synthese von kohlenwasserstoffen aus CO und $\mathrm{H}_{2}$. Chem Eng Tech, 1970, 42 : 1162-1174

42 Cao DB, Wang SG, Li YW, Wang JG, Jiao HJ. What is the product of ketene hydrogenation on $\mathrm{Fe}_{5} \mathrm{C}_{2}(001)$ : oxygenates or hydrocarbons? $J$ Mol Catal A Chem, 2007, 272: 275-287

43 Cao DB, Li YW, Wang JG, Jiao HJ. Chain growth mechanism of Fischer-Tropsch synthesis on $\mathrm{Fe}_{5} \mathrm{C}_{2}(001)$. J Mol Catal A Chem, 2011, 346: 55-69

44 Cao DB, Li YW, Wang JG, Jiao HJ. Adsorption and reaction of surface carbon species on $\mathrm{F}_{\mathrm{e} 5} \mathrm{C}_{2}(001)$. J Phys Chem C, 2008, 112: $14883-14890$

45 Cao DB, Zhang FQ, Li YW, Wang JG, Jiao HJ. Structures and energies of coadsorbed $\mathrm{CO}$ and $\mathrm{H}_{2}$ on $\mathrm{Fe}_{5} \mathrm{C}_{2}(001)$, Fe $\mathrm{C}_{2}(110)$ and $\mathrm{Fe}_{5} \mathrm{C}_{2}$ (100). J Phy Chem B, 2005, 109: 10922-10935

46 Deng LJ, Huo CF, Liu XW, Zhao XH, Li YW, Wang JG, Jiao HJ. Density functional theory study on surface $\mathrm{C}_{x} \mathrm{H}_{y}$ formation from CO activation on $\mathrm{Fe}_{3} \mathrm{C}(100)$. J Phy Chem $C$, 2010, 114: 21585-21592

47 Huo CF, Li YW, Wang JG, Jiao HJ. Surface structure and energeties of hydrogen adsorption on the Fe(111)surface. J Phy Chem B, 2005, 109: 14160-14167

48 Lox ES, Froment GF. Kinetics of the Fischer-Tropsch reaction on a precipitated promoted iron catalyst 1 . Experimental procedure and results. Ind Eng Chem Res, 1993, 32: 61-70

49 Lox ES, Froment GF. Kinetics of the Fischer-Tropsch reaction on a precipitated promoted iron catalyst 2 . Kinetic modeling. Ind Eng Chem Res, 1993, 32: 71-82

50 马文平, 李永旺, 赵玉龙, 徐元源, 周敬来. 工业 $\mathrm{Fe}-\mathrm{Cu}-\mathrm{K}$ 催化剂上费托合成反应动力学(I). 基于机理的动力学模型. 化工学报, 1999, 50: 159-166

51 马文平, 李永旺, 赵玉龙, 周敬来, 钟炳. 工业 $\mathrm{Fe}-\mathrm{Cu}-\mathrm{K}$ 催化剂上费托合成反应动力学(II). 模型筛选与参数估值. 化工学报, 1999 , 50: $167-173$

52 Wang YN, Ma WP, Lu YJ, Yang Jun, Xu YY, Xiang HW, Li YW, Zhao YL, Zhang BJ. Kinetics modeling of Fischer-Tropsch synthesis over an industrial Fe-Cu-K catalyst. Fuel, 2003, 82: 195-213

53 王逸凝, 李永旺, 马文平, 徐元源, 赵玉龙, 张碧江. 费托合成(FTS)动力学模拟一烯烃再吸附. 燃料化学学报, 1999, 27(增刊): $123-128$

54 王逸凝, 李永旺, 徐元源, 赵玉龙, 张碧江. 基于详细机理动力学的费托合成单颗粒催化剂模拟. I. 颗粒模型化与数值计算方法. 催化学报, 2001, 22: 35-39

55 王逸凝, 李永旺, 徐元源, 赵玉龙, 张碧江. 基于详细机理动力学的费托合成单颗粒催化剂模拟 II. 扩散反应行为及活性分布. 催化 学报, 2001, 22: 40-44

56 Wang YN, Xu YY, Xiang HW, Li YW, Zhang BJ. Modeling of catalyst pellets for Fischer-Tropsch synthesis. Ind Eng Chem Res, 2001, 40: 4324-4335

57 吉媛媛, 相宏伟, 李永旺, 徐元源, 钟炳. 超细粒子 Fe-Mn 工业催化剂 F-T 合成集总机理动力学(I). 反应机理与动力学模型描述式. 化工学报, 2005, 56: 1020-1025

58 吉媛媛, 相宏伟, 李永旺, 徐元源, 钟炳. 超细粒子 Fe-Mn 工业催化剂 F-T 合成集总机理动力学(II). 参数估算与模型笁选. 化工学 报, 2005, 56: 1026-1030

59 Yang J, Liu Y, Chang J, Wang YN, Bai L, Xu YY, Xiang HW, Li YW, Zhong B. Detailed kinetics of Fischer-Tropsch synthesis on an industrial Fe-Mn ultrafine particle iron catalyst. Ind Eng Chem Res, 2003, 42: 5066-5090

60 Chang J, Bai L, Teng BT, Zhang RL, Yang J, Xu YY, Xiang HW, Li YW. Kinetic modeling of Fischer-Tropsch synthesis over $\mathrm{Fe}-\mathrm{Cu}-\mathrm{K}-\mathrm{SiO}_{2}$ catalyst in slurry phase reactor. Chem Eng Sci, 2007, 62: 4983-4991

61 Zhang RL, Chang J, Xu YY, Cao LR, Li YW, Zhou JL. Kinetic model of product distribution over Fe catalyst for Fischer-Tropsch synthesis. Energ Fuel, 2009, 23: 4740-4747

62 Zhou LP, Hao X, Gao JH, Yang Y, Wu BS, Xu J, Xu YY, Li YW. Studies and discriminations of the kinetic models for the iron-based Fischer-Tropsch catalytic reaction in a recycle slurry reactor. Energ Fuel, 2011, 25: 52-59 
63 Teng BT, Chang J, Zhang CH, Cao DB, Yang J, Liu Y, Guo XH, Xiang HW, Li YW. A comprehensive kinetics model of Fischer-Tropsch synthesis over an industrial Fe-Mn catalyst. Appl Catal A: Gen, 2006, 301: 39-50

64 Teng BT, Zhang CH, Yang J, Cao DB, Chang J, Xiang HW, Li YW. Oxygenate kinetics in Fischer-Tropsch synthesis over an industrial Fe-Mn catalyst. Fuel, 2005, 84: 791-800

65 Teng BT, Chang J, Yang J, Wang G, Zhang CH, Xu YY, Xiang HW, Li YW. Water gas shift reaction kinetics in Fischer-Tropsch synthesis over an industrial Fe-Mn catalyst. Fuel, 2005, 84: 917-926

66 滕波涛, 常杰, 刘颖, 张成华, 杨骏, 郑洪岩, 张荣乐, 白亮, 相宏伟, 李永旺. 工业 Fe-Mn 催化剂上基于详细反应机理的 F-T 合成 动力学模型 I. 烯烃再吸附动力学研究. 催化学报, 2005, 26: 693-700

67 滕波涛, 常杰, 王刚, 张成华, 刘颖, 郑洪岩, 杨骏, 张荣乐, 白亮, 相宏伟, 李永旺. 工业 Fe-Mn 催化剂上基于详细反应机理的 F-T 合成动力学模型 II. 不同校正方法的动力学模型分析. 催化学报, 2005, 26: 701-706

68 Teng BT, Chang J, Wan HJ, Lu JQ, Zheng SC, Liu Y, Liu Y, Guo XH. A corrected comprehensive kinetic model of Fischer-Tropsch synthesis. Chin J Catal, 2007, 28: 687-695

69 Huo CF, Li YW, Wang JG, Jiao HJ. Insight into $\mathrm{CH}_{4}$ formation in iron-catalyzed Fischer-Tropsch synthesis. J Am Chem Soc, 2009, 131: 14713-14721

70 Huo CF, Wu BS, Gao P, Yang Y, Li YW, Jiao HJ. The mechanism of potassium promoter: enhancing the stability of active surfaces. Angew Chem Int Ed, 2011, 50: 7403-7406

71 Yang T, Wen XD, Li YW, Wang JG, Jiao HJ. Interaction of alkali metals with the $\mathrm{Fe}_{3} \mathrm{O}_{4}(111)$ surface. Surf Sci, 2009, 603: 78-83

72 Zhao S, Liu XW, Huo CF, Li YW, Wang JG, Jiao HJ. Surface morphology of Hagg iron carbide $\left(\gamma-\mathrm{Fe}_{5} \mathrm{C}_{2}\right)$ from ab initioatomistic thermodynamics. J Catal, 2012, 294: 47-53

73 Yang Y, Xiang HW, Zhang RL, Zhong B, Li YW. A highly active and stable Fe-Mn catalyst for slurry Fischer-Tropsch synthesis. Catal Today, 2005, 106: 170-175

74 Yang Y, Xiang HW, Tian L, Wang H, Zhang CH, Tao ZC, Xu YY, Zhong B, Li YW. Structure and Fischer-Tropsch performance of iron-manganese catalyst incorporated with $\mathrm{SiO}_{2}$. Appl Catal A: Gen, 2005, 284: 105-122

75 Yang Y, Xiang HW, Xu YY, Bai L, Li YW. Effect of potassium promoter on precipitated iron-manganese catalyst for Fischer-Tropsch synthesis. Appl Catal A: Gen, 2004, 266: 181-194

76 Zhang CH, Yang Y, Teng BT, Li TZ, Zheng HY, Xiang HW, Li YW. Study of an iron-manganese Fischer-Tropsch synthesis catalyst promoted with copper. J Catal, 2006, 237: 405-415

77 Suo HY, Wang SG, Zhang CH, Xu J, Wu BS, Yang Y, Xiang HW, Li YW. Chemical and structural effects of silica in iron-based Fischer-Tropsch synthesis catalysts. $J$ Catal, 2012, 286: 111-123

78 Qing M, Yang Y, Wu BS, Xu J, Zhang CH, Gao P, Li YW. Modification of $\mathrm{Fe}_{-} \mathrm{SiO}_{2}$ interaction with zirconia for iron-based Fischer-Tropsch catalysts. J Catal, 2011, 279: 111-122

79 Ding MY, Yang Y, Wu BS, Wang TJ, Ma LL, Xiang HW, Li YW. Transformation of carbonaceous species and its influence on catalytic performance for iron-based Fischer-Tropsch synthesis catalyst. J Mol Catal A Chem, 2011, 351: 165-173

80 Ding MY, Yang Y, Wu BS, Xu J, Zhang CH, Xiang HW, Li YW. Study of phase transformation and catalytic performance on precipitated iron-based catalyst for Fischer-Tropsch synthesis. J Mol Catal A Chem, 2009, 303: 65-71

81 Hou WJ, Wu BS, Yang Y, Hao QL, Tian L, Xiang HW, Li YW. Effect of $\mathrm{SiO}_{2}$ content on iron-based catalysts for slurry Fischer-Tropsch synthesis. Fuel Proc Tech, 2008, 89: 284-291

82 Tao ZC, Yang Y, Wan HJ, Li TZ, An X, Xiang HW, Li YW. Effect of manganese on a potassium-promoted iron-based Fischer-Tropsch synthesis catalyst. Catal Lett, 2007, 114: 161-168

83 Hou WJ, Wu BS, An X, Li TZ, Tao ZC, Zheng HY, Xiang HW, Li YW. Effect of the ratio of precipitated $\mathrm{SiO}_{2}$ to binder $\mathrm{SiO}_{2}$ on iron-based catalysts for Fischer-Tropsch synthesis. Catal Lett, 2007, 119: 353-360

84 Yang J, Sun YC, Tang Y, Liu Y, Wang HL, Tian L, Wang H, Zhang Zh X, Xiang HW, Li YW. Effect of magnesium promoter on iron-based catalyst for Fischer-Tropsch synthesis. J Mol Catal A Chem, 2006, 245: 26-36

85 Yang T, Wen XD, Huo CF, Li YW, Wang JG, Jiao HJ. Carburization of the $\mathrm{Fe}_{3} \mathrm{O}_{4}(111)$ surface. J Phys Chem C, 2008, 112: 6372-6379

86 Ding MY, Yang Y, Xu J, Tao ZC, Wang HL, Wang H, Xiang HW, Li YW. Effect of reduction pressure on precipitated potassium promoted iron-manganese catalyst for Fischer-Tropsch synthesis. Appl Catal A: Gen, 2008, 345: 176-184

87 Hao QL, Liu FX, Wang H, Chang J, Zhang CH, Bai L, Xiang HW, Li YW, Yi F, Xu BF. Effect of reduction temperature on a spray-dried iron-based catalyst for slurry Fischer-Tropsch synthesis. J Mol Catal A Chem, 2007, 261: 104-111

88 Wang H, Yang Y, Wu BS, Xu J, Ding MY, Wang HL, Fan WH, Xiang HW, Li YW. Hydrogen reduction kinetics modeling of a 
precipitated iron Fischer-Tropsch catalyst. J Mol Catal A Chem, 2009, 308: 96-107

89 Ding MY, Yang Y, Wu BS, Wang TJ, Xiang HW, Li YW. Effect of reducing agents on microstructure and catalytic performance of precipitated iron-manganese catalyst for Fischer-Tropsch synthesis. Fuel Proc Tech, 2011, 92: 2353-2359

90 杨勇，吴宝山，许健，相宏伟，李永旺.一种连续化制备金属氧化物材料和催化剂的方法. 中国专利：ZL200810186615.2, 2008-11-25

91 相宏伟，杨勇，陶智超，田否，李永旺. 一种铁锰费托合成催化剂及其制法. 中国专利, ZL200410012191.X, 2004-03-16

92 李永旺, 相宏伟, 吴宝山, 钟炳. 一种费托合成用铁基催化剂及其制备方法. 中国专利, ZL 01120417.6, 2001-07-12

93 相宏伟, 张志新, 李国辉, 李永旺. 一种微球状费托合成催化剂的制备方法. 中国专利, ZL01120416.8, 2001-07-12

94 Li YW, Hao X, Cao LR, Wang JS, Liu DX, Bai L. A gas-liquid-solid three-phase suspension bed reactor for Fischer-Tropsch synthesis and the use thereof. Australia Patent, AU2007359708, 2009-10-06

95 王晋生, 郝栩, 刘东勋, 白亮, 曹立仁, 李永旺. 用于费-托合成的气-液-固三相悬浮床反应器及其应用. 中国专利, ZL200710161575.1, 2007-09-29

96 Wang G, Wang YN, Yang J, Xu YY, Li YW. Modeling analysis of the Fischer-Tropsch synthesis in a stirred-tank slurry reactor. Ind Eng Chem Res, 2004, 43: 2330-2336

97 Wang Y, Fan W, Liu Y, Zeng ZY, Hao X, Chang M, Zhang CH, Xu YY, Xiang HW, Li YW. Modeling of the Fischer-Tropsch synthesis in slurry bubble column reactors. Chem Eng Proc, 2008, 47: 222-228

98 Wang YN, Li YW, Zhao YL, Zhang BJ. Modeling of Fischer-Tropsch synthesis in bubble column slurry reactors: numerical analysis. $J$ Offuel Chem Echhno, 1999, 27: 193-202

99 焚伟, 郝栩, 相宏伟, 徐元源, 李永旺. F-T 合成反应器汽包换热系统动态响应研究. 现代化工, 2010, 30: 64-68

100 任杰, 张怀科, 李永旺. F-T 合成油品加工技术的研究进展. 燃料化学学报, 2009, 37: 769-776

101 任杰, 李永旺, 王峰, 王雪峰, 姜建卫. 一种用于非硫化费托合成油品加氢转化的工艺.中国专利, ZL200510012800.6, 2005-09-08

102 任杰, 相宏伟, 曹立仁, 孙予罕, 李永旺. 费-托合成油的加氢处理方法. 中国专利, ZL200710065310.1, 2007-04-11

103 任杰, 相宏伟, 曹立仁, 孙予罕, 李永旺. 费-托合成油的加氢处理工艺. 中国专利, ZL200710065309.9, 2007-04-11

104 董立华, 郝栩, 曹立仁, 李永旺. 费托合成油品脱酸和含氧化合物过程模拟. 煤炭转化, 2009, 32: 14-17

105 黄勇成, 李永旺, 任杰, 周龙保. F-T 柴油在直喷式柴油机中燃烧与排放特性的研究. 燃料化学学报, 2005, 23: 492-496

106 许丽恒. 费托合成蜡加氢裂解动力学研究. 硕士学位论文. 太原: 中国科学院山西煤炭化学研究所, 2013. 1-111

107 许丽恒, 高军虎, 郝栩, 李永旺. 费托合成蜡加氢裂化工艺条件的研究. 石油炼制与化工, 2013, 44: 85-89

108 中科合成油技术有限公司. 煤基合成液体燃料工业设计软件 1.0. 版权登记号: 2006SR12354, 国家版权局, 2006 年

109 于戈文, 徐元源, 郝栩, 李永旺. 不同煤气化过程的 FT 合成油-电多联产模拟计算. 过程工程学报, 2009, 9: 545-551

110 Hao X, Elissa DM, Xu YY, Wang YN, Chang J, Li YW. Simulation analysis of a GTL process using ASPEN plus. Chem Eng Tech, 2008, 31: 188-196

111 Yu GW, Xu YY, Hao X, Li YW, Liu GQ. Process analysis for polygeneration of Fischer-Tropsch liquids and power with $\mathrm{CO}_{2}$ capture based on coal gasification. Fuel, 2010, 89: 1070-1076

112 于戈文, 徐元源, 郝栩, 李永旺. 以 Shell 气化为气头的二甲醚-电多联产系统分析. 现代化工, 2009, 29: 62-66

113 国家发改委, 国家能源局. 煤炭深加工示范项目规划. 2012

114 王文. 2016 年全国煤制油项目产能将达 1600 万 t. 煤炭加工与综合利用, 2013, 4: 80

115 全国煤化工信息站. 煤制油、煤制气指导意见将于近期正式发布一《关于有序推进煤制油示范项目建设的指导意见》. 煤化工, 2014, 42: $70-71$

116 李慧. 煤制油产业三问: 未来-产业化-环保. 化工管理, 2013, 10: 45-46

117 张兴刚. 煤制油技术: 能源替代殊途同归. 中国石油和化工, 2013, 10: 16-18

118 崔民利, 黄剑薇, 郝栩, 曹立仁, 李永旺. 含碳固体燃料的分级液化方法和用于该方法的三相悬浮床反应器. 中国专利, ZL200910178131.8, 2009-10-09 


\title{
Indirect coal-to-liquids technology from fundamental research to commercialization
}

\author{
XIANG HongWei ${ }^{1,2}$, YANG Yong ${ }^{1,2}$, LI YongWang ${ }^{1,2 *}$ \\ 1 State Key Laboratory of Coal Conversion, Institute of Coal Chemistry, Chinese Academy of Sciences, Taiyuan 030001, China \\ 2 National Energy Research Center for Coal-based Clean Fuels, Synfuels CHINA Co., Ltd., Beijing 101407, China \\ *Corresponding author (email: ywl@ sxicc.ac.cn)
}

\begin{abstract}
China needs to independently develop indirect coal-to-liquids(CTL) technology for commercialization, which can relieve the tense situation of oil supply and ensure the economic sustainable development. Currently, China has successfully run CTL demonstration plant and achieved the industrially proven technology of FischerTropsch catalyst and synthesis reactor, and has been designing and setting up million ton scale CTL commercial plant. The paper briefly introduces the progress of indirect CTL technology over the world, reviews the research progress from basic research to engineering technology in China's coal indirect liquefaction process, including Fischer-Tropsch synthesis mechanism, catalyst preparation, reaction kinetics, industrial reactor design, system process integration and synfuels processing, highlights the key basic research and engineering problems for the construction of CTL industry with an outlook for the future, and puts forward the challenges and countermeasures in China.
\end{abstract}

Keywords: indirect coal-to-liquids, Fischer-Tropsch synthesis, Fe-based catalyst, slurry reactor 